\title{
Initiation of backward erosion piping in uniform sands
}

\author{
V. M. VAN BEEK*†, A. BEZUIJEN $\$$, J. B. SELLMEIJER* and F. B. J. BARENDS*†
}

\begin{abstract}
The process of backward erosion piping poses a threat to dams and dikes on foundations of nonplastic sands and silts. The available models for design and predictions focus predominantly on the progression of the pipe. However, sand boils in the field will occur as a result of the initiation of sand transport. Although criteria are available for predicting sand boiling and heaving in columns, there is no model describing the initiation of piping in situations where the exit flow is not uniform, as is the case in most backward erosion experiments and situations in the field. This study compared laboratory experiments in which the process of initiation leads directly to failure with analytical and numerical groundwater flow calculations and heave criteria. The aim was to develop a model for the onset of pipe formation. It emerged that the sand bed needs to be fluidised over a distance of at least 20 times the grain diameter from the toe of the structure for a pipe to initiate. The proposed model explains the scale effects of grain size and configuration on a critical gradient. This approach clarifies the processes governing pipe initiation and progression and it can be used to establish a conservative estimate of the critical head in uniform sands, which is essential for laboratory work on this topic and for the appraisal of sand boils in practice.
\end{abstract}

KEYWORDS: design; embankments; numerical modelling

\section{INTRODUCTION}

Internal erosion, the removal of particles from within an embankment or its foundation by seepage flow, is considered to be one of the main causes of dam failures (Foster et al., 2000). An evaluation by Richards \& Reddy (2007) showed that up to one-third of all internal erosion failures can be attributed to backward erosion and suffusion. Backward erosion is a form of internal erosion that may occur in the non-plastic silts or sands of a foundation or core of a dam or dike. A distinction is made between pipe formation in a near-horizontal direction below a cohesive layer - this is known as backward erosion piping - and global backward erosion, where near-vertical pipes form in non-plastic embankment cores (ICOLD, 2013). This article focuses on backward erosion piping in the foundations of embankments, in which the erosion process is essentially horizontal and in which particles are detached and carried away to an unfiltered exit, resulting in a hollow space that forms in the direction opposite to the flow.

If the pervious layer downstream of the structure is not protected by filters or intact blanket layers, water flow through the foundation can cause particle transport downstream of the embankment, resulting in the formation of shallow pipes at the interface of the granular layer and an overlying cohesive layer (Fig. 1). These shallow pipes develop in the opposite direction to the water flow until the upstream water is reached, establishing a connection between the upstream and downstream water levels. The increased flow through the connecting pipes leads to ongoing erosion and finally, given enough time, to the collapse and failure of the structure.

Manuscript received 6 December 2013; revised manuscript accepted 23 October 2014.

Discussion on this paper is welcomed by the editor.

* Unit Geo-Engineering, Deltares, Delft, the Netherlands.

$\dagger$ Faculty of Civil Engineering and Geosciences, Delft University of Technology, Delft, the Netherlands.

† Department Civil Engineering, Ghent University, Ghent, Belgium; also Unit Geo-Engineering, Deltares, Delft, the Netherlands.
The conditions required for backward erosion are often present in delta areas, where uniform sandy aquifers are covered by cohesive soft soils and embankments to prevent the flooding of the hinterland. Backward erosion has been reported as an important failure mechanism in river dikes in the Netherlands, China and the USA (Cao, 1994; Mansur et al., 2000; ENW, 2010).

Preventive steps such as filters or cut-offs can be included in embankments with a limited length, such as dams. However, this is not feasible along the entire length of river dikes. Here, prediction tools are vital in the assessment of flood risks and in decisions about where reinforcement is required.

\section{Literature review}

Prediction tools for backward erosion have been developed since the early 1900s and improvements are still being made. Some of these models rely to a large extent on case histories or laboratory and full-scale experiments, and they are empirical or semi-empirical in nature. Others are based on physical concepts and have been calibrated using experiments.

Bligh (1910) was the first to establish a relationship between the average hydraulic gradient $(H / L)$ across the structure, the soil type and the occurrence of internal erosion on the basis of data derived from specific cases of piping. This resulted in an empirical formulation to determine the critical gradient. The critical gradient $\left(H_{\mathrm{c}} / L\right)$ is defined as the ratio between the critical head ( $H_{\mathrm{c}}$, the head that leads to ongoing erosion) and seepage length ( $L$, the shortest distance between the upstream and downstream levels), as illustrated in Fig. 1. Lane (1935) contributed to this empirical formulation by adjusting the weight factor for vertical sections in the seepage path. Although the approaches were entirely empirical, it was already realised at that time that groundwater flow determines the magnitude and distribution of seepage forces near the toe of the structure and therefore has a considerable influence on the critical gradient. Accordingly, it must be modelled correctly, as noted by Casagrande in his response to Lane (1935) referring to Terzaghi (1922). 


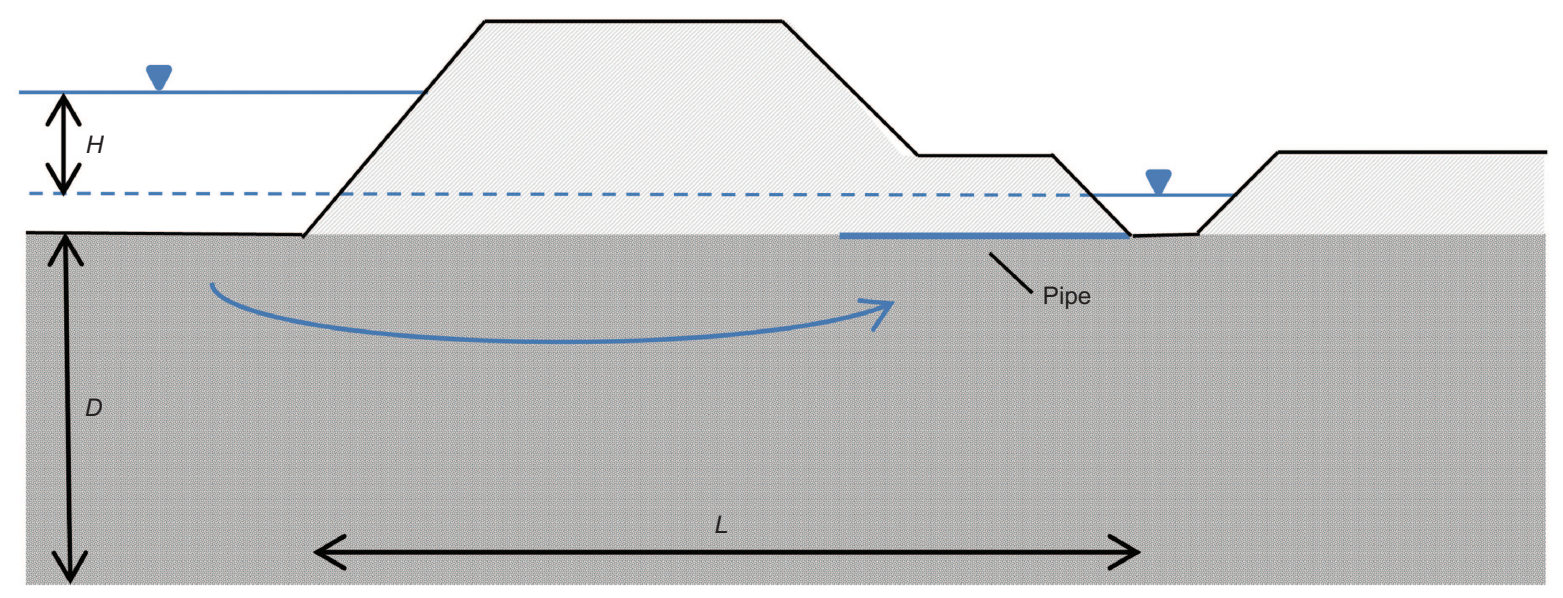

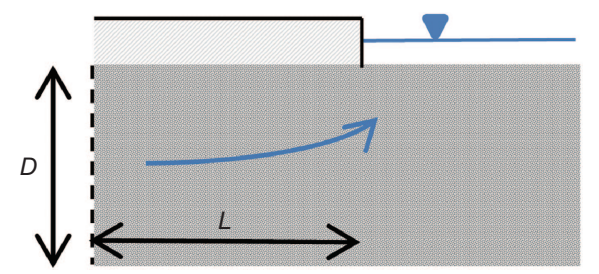

(a)

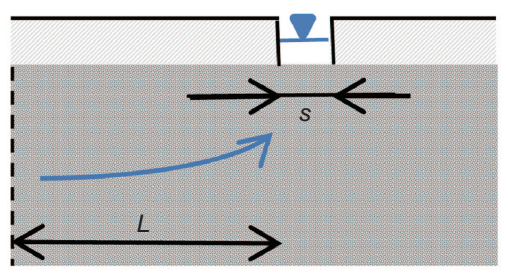

(b)

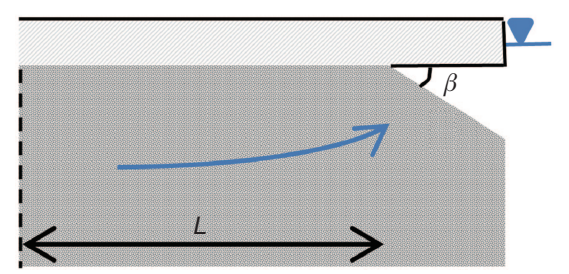

(c)

Fig. 1. Schematised embankment with sandy or silty foundation and experimental configurations with upstream filter and different exit types: (a) plane, (b) ditch, (c) slope, simulating the embankment and its foundations

In the 1980s De Wit (1984) completed a major research programme with the aim of clarifying backward erosion. A wide variety of laboratory experiments were performed, with different exit types, sand types, scales and porosities, to determine the critical gradient. De Wit (1984) explained observed scale effects for the critical gradient $\left(H_{\mathrm{c}} / L\right)$ using analytical equations for groundwater flow and the local horizontal gradient below the dike near the toe. Sand type, porosity and exit type were found to be major determinants of the critical gradient. At the same time, a German research group (Miesel, 1978; Hanses, 1985; Müller-Kirchenbauer et al., 1993) conducted an extensive study of the erosion process. Pipe progression was modelled numerically (using potential theory analysis) for different scales and for multilayer configurations. Sellmeijer (1988) combined groundwater flow equations with equations for the micro-scale processes in the pipe (grain equilibrium and Posseuille flow). It was assumed that a pipe continues to grow when the seepage force on the grains at the bottom of the pipe exceeds the stabilising forces. For each pipe length, the 'equilibrium head drop', which is the head drop across the structure at which the grains in the pipe are in the limit equilibrium state, was calculated by solving equations for groundwater flow, pipe flow and grain equilibrium (Sellmeijer, 1988). The maximum equilibrium head found in this way is referred to as the 'critical head' for the progression of the pipe because ongoing erosion will occur when this maximum head is exceeded. The model was calibrated using large-scale experiments in the Delta flume (Silvis, 1991) and a practical rule was derived for a standard configuration (Weijers \& Sellmeijer, 1993). This rule was recently adapted on the basis of the results of additional experiments (Sellmeijer et al., 2011). Deviating configurations can be analysed using a finite-element method (FEM) code in which the model has been integrated (Sellmeijer, 2006). The model is used for safety assessments in the Netherlands (TAW, 1999). In parallel with this approach, Schmertmann (2000) developed a design method based on flow nets and empirical factors derived using experimental results from Pietrus (1981), Townsend et al. (1988) and De Wit (1984). He found that the uniformity coefficient was highly significant (the critical gradient increased in line with increases in the uniformity coefficient). It should be pointed out that this conclusion was based on a limited number of tests on sands with larger uniformity coefficients, including some tests on internally unstable soils.

The models that predict the critical gradient by including the presence of a pipe assume that, after initiation, the pipe does not get longer and that there is therefore 'equilibrium'. Accordingly, the gradient is calculated that allows the pipe to progress in the upstream direction. The implicit assumption is that the onset of pipe development, or 'initiation', requires a lower head drop than the 'progression' of the pipe.

However, this assumption is not valid for all experiments. In some experiments, equilibrium is observed after pipe formation and an increase in the head drop is needed for the pipe to lengthen (Miesel, 1978; Hanses, 1985; Silvis, 1991; Müller-Kirchenbauer et al., 1993, some of the experiments by Pietrus, 1981; Townsend et al., 1988; and Van Beek et al., 2011). In these experiments, the presence of the pipe affects the continuation of pipe development. In other experiments, no equilibrium is observed: the pipe develops continuously towards the upstream side without any further increase in head (small-scale experiments by Van Beek et al. (2011), experiments by De Wit (1984), and some of the experiments by Pietrus (1981) and Townsend et al. (1988)). Essentially, in the second type of experiment, the critical gradient $\left(H_{\mathrm{c}} / L\right)$ is determined by the initiation of the pipe rather than the progression of the pipe. Since there is no equilibrium during pipe development, it must be concluded that the initiation of the pipe in these experiments requires a larger head than the head required for the progression of the pipe (see also section 'Initiation and progression'). The determination of the critical head by initiation or progression proves to be dependent on the type of exit (see Fig. 1), as will be explained later. 


\section{Terminology}

To maintain clarity, notations have been used for the different heads and gradients in this paper. The critical gradient $\left(H_{\mathrm{c}} / L\right)$ is defined as the average gradient across the structure that leads to ongoing erosion. This critical gradient can be determined by two processes: initiation or progression. In all experiments, the pipe had to be initiated, and this event occurred at the initiation head $\left(H_{\mathrm{i}}\right)$ or average initiation gradient $\left(H_{\mathrm{i}} / L\right)$. In some experiments, pipe equilibrium was then observed, and the head needed to be raised until the pipe progressed in the upstream direction. The head required for pipe progression, assuming the presence of a short pipe, is referred to as $H_{\mathrm{p}}$, and the corresponding average gradient for progression is $H_{\mathrm{p}} / L$. In other experiments, no equilibrium was observed after the initiation of the pipe and the pipe progressed in the upstream direction without any further increase in the head. In these experiments the critical gradient was determined by the process of initiation and the progression gradient $\left(H_{\mathrm{p}} / L\right)$ remains in most cases unknown since the progression gradient had already been exceeded at the point of initiation.

\section{Initiation and progression}

The difference between initiation and progression has been confirmed in small-scale experiments described in Van Beek et al. (2013) in which the head drops required for progression $\left(H_{\mathrm{p}}\right)$ and initiation $\left(H_{\mathrm{i}}\right)$ were studied separately. In an experiment with a slope-type exit (Fig. 1), in which equilibrium in pipe growth is normally not observed, the head drop was raised until the pipe initiated and developed over a certain distance. Usually the pipe continues to grow until failure. In this experiment, the head drop was reduced to zero when the pipe was still short and then raised again in steps until piping continued. This process was repeated at several pipe lengths. It emerged that the head required for pipe progression $\left(H_{\mathrm{p}}\right)$ was lower than the head required for pipe initiation $\left(H_{\mathrm{i}}\right)$. A second experiment, which was identical except that the exit was now of the hole type, and in which the head drop required for initiation $\left(H_{\mathrm{i}}\right)$ was considerably lower due to the concentration of flow lines around the exit, showed that the progression of piping in these conditions requires a larger head drop than initiation. It is clear that the type of exit is one of the parameters that determines whether progression or initiation dominates the piping process and corresponding critical head.

\section{Objectives}

Although initiation and progression are considered different steps in the process of internal erosion (Foster \& Fell, 1999), this difference has not been acknowledged by the prediction models for backward erosion described here. Most models developed to predict the critical head for backward erosion piping included both initiation-dominated experiments and progression-dominated experiments in calibration and validation, even though pipe initiation and pipe progression are different mechanisms (Sellmeijer, 1988; Schmertmann, 2000; Sellmeijer et al., 2011). An exception to this is the work by Richards \& Reddy (2012), who performed experiments with the explicit aim of modelling piping initiation using a triaxial set-up. They found that exit seepage velocity was an important parameter for pipe initiation. However, the authors of this paper believe that the local exit gradient causes the initiation of erosion, rather than the exit velocity. Furthermore, their approach is currently applicable to the triaxial set-up only, since the exit velocity near the toe of a water-retaining structure depends very much on the location and the exit configuration.
The present study developed a prediction model for pipe initiation with different configurations. It puts forward suggestions to determine when initiation or progression is likely to be the dominant determinant of the height of the critical head. This information is not only essential for experimental work studying backward erosion and the development of prediction models for backward erosion, it will also give practical information required to decide whether it is likely that a sand boil indicates ongoing erosion, which may result in failure over time.

\section{EXPERIMENTS AVAILABLE FOR ANALYSIS}

Both initiation-dominated and progression-dominated experiments are suitable for the analysis of the initiation of piping since the initiation gradient $\left(H_{\mathrm{i}} / L\right)$ can be recorded in both types of experiment. However, the head at which the pipe is initiated $\left(H_{\mathrm{i}}\right)$ is not always recorded in progressiondominated experiments, focusing exclusively on the critical gradient $\left(H_{\mathrm{c}} / L\right)$. As a result, the analysis here mainly includes experiments in which no equilibrium is observed after the initial sand transport, unless the initiation gradient has been recorded. The experiments in which the critical head is determined by the process of initiation are typically smallscale experiments with a relatively large exit area, as described in Van Beek et al. (2013). New small-scale experiments were performed to supplement the work of De Wit (1984) and they are presented in this paper.

Both the experiments by De Wit (1984) and Van Beek et al. (2011) were performed at different scales with different sand types and porosities. The characteristics of the sand types used in the experiments are listed in Table 1. The observations reported by De Wit (1984) for plane-type and ditch-type experiments (characteristics summarised in Table 2 and later in Table 4) are as follows.

(a) Expansion of the sand bed: at the toe of the clay layer the sand starts to expand, creating an elevated area with an estimated height of $1 \mathrm{~mm}$ and an estimated width of approximately $15-20 \mathrm{~mm}$. This expansion is most clearly observed in experiments with fine sands and it is not seen in experiments with coarse sand.

(b) Small holes due to the local wash-out of grains near the toe of the embankment.

(c) Small sand boils in which sand is lifted and deposited, no sand transport.

(d) Increased boiling of sand in sand boils, with no sand transport.

(e) A sand boil starts to deposit sand and grows in size. A crater is formed. The process continues until there is a breach.

This description indicates that the head at which the pipe reaches the upstream side $\left(H_{\mathrm{c}}\right)$ is equal to the head at which the pipe is initiated $\left(H_{\mathrm{i}}\right)$. In most experiments, the required head for sand boiling $(d), H_{\mathrm{b}}$ here, is more or less equal to the head for sand transport $(e)$. However, in some experiments, sand boils occur at a much lower head drop $\left(H_{\mathrm{b}}\right)$ than

Table 1. Sand characteristics

\begin{tabular}{|c|c|c|c|c|}
\hline Sand type & $d_{60} / d_{10}$ & $d_{50}: \mathrm{mm}$ & $n_{\min }$ & $n_{\max }$ \\
\hline Dune sand & $1 \cdot 48$ & $0 \cdot 190$ & $0 \cdot 341$ & $0 \cdot 449$ \\
\hline Beach sand & $1 \cdot 33$ & $0 \cdot 200$ & $0 \cdot 330$ & 0.447 \\
\hline River sand & $2 \cdot 30$ & $0 \cdot 400$ & $0 \cdot 317$ & $0 \cdot 400$ \\
\hline Sieved river sand & $2 \cdot 10$ & $0 \cdot 365$ & $0 \cdot 315$ & 0.404 \\
\hline Coarse sand & $3 \cdot 85$ & $0 \cdot 750$ & $0 \cdot 235$ & $0 \cdot 400$ \\
\hline Enschedé sand & $1 \cdot 60$ & $0 \cdot 380$ & $0 \cdot 320$ & $0 \cdot 411$ \\
\hline Baskarp sand & $1 \cdot 60$ & $0 \cdot 132$ & $0 \cdot 340$ & 0.469 \\
\hline
\end{tabular}


Table 2. Plane-type experiments (De Wit, 1984)

\begin{tabular}{|c|c|c|c|c|c|c|c|c|}
\hline Test no. & Sand type & $L: \mathrm{m}$ & $D: \mathrm{m}$ & $n$ & $k: \mathrm{m} / \mathrm{s}$ & $i_{\mathrm{c}}$ & $H_{\mathrm{i}}: \mathrm{m}$ & $H_{\mathrm{c}}: \mathrm{m}$ \\
\hline 220880-I-1 & Dune sand & $0 \cdot 8$ & $0 \cdot 5$ & $0 \cdot 357$ & $1 \cdot 1 \times 10^{-4}$ & $1 \cdot 061$ & $0 \cdot 330$ & $0 \cdot 330$ \\
\hline 220880-I-2 & Dune sand & $0 \cdot 8$ & $0 \cdot 5$ & $0 \cdot 352$ & $8.9 \times 10^{-5}$ & 1.069 & $0 \cdot 364$ & $0 \cdot 364$ \\
\hline 220880-I-3 & Dune sand & $0 \cdot 8$ & $0 \cdot 5$ & $0 \cdot 353$ & $1 \cdot 1 \times 10^{-4}$ & 1.068 & $0 \cdot 331$ & $0 \cdot 331$ \\
\hline 220880-I-4 & Dune sand & $0 \cdot 8$ & $0 \cdot 5$ & $0 \cdot 370$ & $1.5 \times 10^{-4}$ & $1 \cdot 040$ & 0.239 & $0 \cdot 239$ \\
\hline 220880-I-5 & Dune sand & $0 \cdot 8$ & $0 \cdot 5$ & $0 \cdot 380$ & $1.5 \times 10^{-4}$ & 1.023 & 0.269 & $0 \cdot 269$ \\
\hline $220880-\mathrm{I}-6$ & Dune sand & $0 \cdot 8$ & $0 \cdot 5$ & $0 \cdot 362$ & $1 \cdot 8 \times 10^{-4}$ & $1 \cdot 053$ & 0.272 & $0 \cdot 272$ \\
\hline $220880-\mathrm{I}-7$ & Dune sand & $0 \cdot 8$ & $0 \cdot 5$ & $0 \cdot 392$ & $2 \cdot 5 \times 10^{-4}$ & $1 \cdot 003$ & $0 \cdot 201$ & $0 \cdot 201$ \\
\hline $220880-\mathrm{I}-8$ & Dune sand & $0 \cdot 8$ & 0.5 & 0.409 & $2 \cdot 7 \times 10^{-4}$ & 0.975 & $0 \cdot 166$ & $0 \cdot 166$ \\
\hline 220880-I-9 & Dune sand & $0 \cdot 8$ & 0.5 & $0 \cdot 400$ & $3 \cdot 3 \times 10^{-4}$ & 0.990 & $0 \cdot 222$ & 0.222 \\
\hline 220880-II-1 & River sand & $0 \cdot 8$ & 0.5 & $0 \cdot 330$ & $3.7 \times 10^{-4}$ & $1 \cdot 106$ & $0 \cdot 302$ & $0 \cdot 302$ \\
\hline 220880-II-2 & River sand & $0 \cdot 8$ & $0 \cdot 5$ & $0 \cdot 338$ & $3.9 \times 10^{-4}$ & 1.092 & 0.450 & 0.450 \\
\hline 220880-II-3 & River sand & $0 \cdot 8$ & 0.5 & $0 \cdot 350$ & $5 \cdot 2 \times 10^{-4}$ & 1.073 & $0 \cdot 300$ & $0 \cdot 300$ \\
\hline 220880-II-4 & River sand & $0 \cdot 8$ & $0 \cdot 5$ & $0 \cdot 360$ & $6 \cdot 1 \times 10^{-4}$ & 1.056 & $0 \cdot 445$ & $0 \cdot 445$ \\
\hline 220880-II-5 & River sand & $0 \cdot 8$ & $0 \cdot 5$ & $0 \cdot 370$ & $6.6 \times 10^{-4}$ & $1 \cdot 040$ & $0 \cdot 340$ & $0 \cdot 340$ \\
\hline $220880-I I-6$ & River sand & $0 \cdot 8$ & $0 \cdot 5$ & $0 \cdot 380$ & $7 \cdot 5 \times 10^{-4}$ & $1 \cdot 023$ & $0 \cdot 225$ & $0 \cdot 225$ \\
\hline 220880-III-1 & Sieved river sand & $0 \cdot 8$ & $0 \cdot 5$ & $0 \cdot 340$ & $3.7 \times 10^{-4}$ & 1.089 & $0 \cdot 300$ & $0 \cdot 300$ \\
\hline 220880-III-2 & Sieved river sand & $0 \cdot 8$ & $0 \cdot 5$ & $0 \cdot 350$ & $3 \cdot 7 \times 10^{-4}$ & 1.073 & $0 \cdot 392$ & $0 \cdot 392$ \\
\hline 220880-III-3 & Sieved river sand & $0 \cdot 8$ & $0 \cdot 5$ & $0 \cdot 359$ & $3 \cdot 8 \times 10^{-4}$ & $1 \cdot 058$ & $0 \cdot 364$ & $0 \cdot 364$ \\
\hline 220880-III-4 & Sieved river sand & $0 \cdot 8$ & $0 \cdot 5$ & $0 \cdot 370$ & $4.6 \times 10^{-4}$ & $1 \cdot 040$ & $0 \cdot 284$ & $0 \cdot 284$ \\
\hline 220880-III-5 & Sieved river sand & $0 \cdot 8$ & $0 \cdot 5$ & $0 \cdot 380$ & $5 \cdot 3 \times 10^{-4}$ & $1 \cdot 023$ & $0 \cdot 322$ & $0 \cdot 322$ \\
\hline 220880-III-6 & Sieved river sand & $0 \cdot 8$ & $0 \cdot 5$ & $0 \cdot 390$ & $6.9 \times 10^{-4}$ & $1 \cdot 007$ & $0 \cdot 202$ & $0 \cdot 202$ \\
\hline 220880-IV-1 & Dune sand & $2 \cdot 4$ & $1 \cdot 5$ & $0 \cdot 350$ & $1.4 \times 10^{-4}$ & $1 \cdot 073$ & $0 \cdot 838$ & $0 \cdot 838$ \\
\hline 220880-IV-2 & Dune sand & $2 \cdot 4$ & $1 \cdot 5$ & $0 \cdot 360$ & $1.7 \times 10^{-4}$ & $1 \cdot 056$ & $0 \cdot 374$ & $0 \cdot 374$ \\
\hline 220880-IV-3 & Dune sand & $2 \cdot 4$ & $1 \cdot 5$ & $0 \cdot 370$ & $1.9 \times 10^{-4}$ & $1 \cdot 040$ & 0.409 & 0.409 \\
\hline $220880-\mathrm{V}-1$ & Beach sand & $0 \cdot 8$ & $0 \cdot 5$ & $0 \cdot 340$ & $1.6 \times 10^{-4}$ & 1.089 & $0 \cdot 266$ & $0 \cdot 266$ \\
\hline $220880-\mathrm{V}-2$ & Beach sand & $0 \cdot 8$ & $0 \cdot 5$ & $0 \cdot 350$ & $1.9 \times 10^{-4}$ & 1.073 & $0 \cdot 303$ & $0 \cdot 303$ \\
\hline $220880-V-3$ & Beach sand & $0 \cdot 8$ & $0 \cdot 5$ & $0 \cdot 360$ & $2 \cdot 1 \times 10^{-4}$ & 1.056 & 0.234 & 0.234 \\
\hline $220880-V-4$ & Beach sand & $0 \cdot 8$ & $0 \cdot 5$ & $0 \cdot 370$ & $2 \cdot 6 \times 10^{-4}$ & $1 \cdot 040$ & $0 \cdot 244$ & $0 \cdot 244$ \\
\hline $220880-V-5$ & Beach sand & $0 \cdot 8$ & $0 \cdot 5$ & $0 \cdot 380$ & $2 \cdot 4 \times 10^{-4}$ & $1 \cdot 023$ & $0 \cdot 208$ & $0 \cdot 208$ \\
\hline $220880-V-6$ & Beach sand & $0 \cdot 8$ & $0 \cdot 5$ & $0 \cdot 390$ & $2.9 \times 10^{-4}$ & 1.007 & $0 \cdot 250$ & $0 \cdot 250$ \\
\hline $220880-V-7$ & Beach sand & $0 \cdot 8$ & $0 \cdot 5$ & $0 \cdot 400$ & $3.4 \times 10^{-4}$ & 0.990 & $0 \cdot 244$ & $0 \cdot 244$ \\
\hline 220880-VI-1 & Beach sand & $2 \cdot 4$ & $1 \cdot 5$ & $0 \cdot 344$ & $2 \cdot 0 \times 10^{-4}$ & $1 \cdot 082$ & $0 \cdot 415$ & 0.415 \\
\hline 220880-VI-2 & Beach sand & $2 \cdot 4$ & $1 \cdot 5$ & $0 \cdot 350$ & $1.9 \times 10^{-4}$ & 1.073 & $0 \cdot 352$ & $0 \cdot 352$ \\
\hline 220880-VI-3 & Beach sand & $2 \cdot 4$ & $1 \cdot 5$ & $0 \cdot 368$ & $2.4 \times 10^{-4}$ & $1 \cdot 043$ & $0 \cdot 414$ & 0.414 \\
\hline 220880-VI-4 & Beach sand & $2 \cdot 4$ & $1 \cdot 5$ & $0 \cdot 370$ & $2 \cdot 3 \times 10^{-4}$ & $1 \cdot 040$ & $0 \cdot 444$ & $0 \cdot 444$ \\
\hline 220880-VI-5 & Beach sand & $2 \cdot 4$ & $1 \cdot 5$ & $0 \cdot 380$ & $2.9 \times 10^{-4}$ & $1 \cdot 023$ & $0 \cdot 360$ & $0 \cdot 360$ \\
\hline 220880-VI-6 & Beach sand & $2 \cdot 4$ & $1 \cdot 5$ & $0 \cdot 390$ & $3.0 \times 10^{-4}$ & $1 \cdot 007$ & $0 \cdot 381$ & $0 \cdot 381$ \\
\hline 220880-VI-7 & Beach sand & $2 \cdot 4$ & $1 \cdot 5$ & $0 \cdot 400$ & $3.5 \times 10^{-4}$ & 0.990 & $0 \cdot 285$ & $0 \cdot 285$ \\
\hline 220880-VII-1 & Beach sand & $0 \cdot 8$ & 0.5 & $0 \cdot 336$ & $1.4 \times 10^{-4}$ & 1.096 & $0 \cdot 280$ & $0 \cdot 280$ \\
\hline 220880-VII-2 & Beach sand & $0 \cdot 8$ & 0.5 & $0 \cdot 350$ & $1.8 \times 10^{-4}$ & 1.073 & $0 \cdot 241$ & $0 \cdot 241$ \\
\hline 220880-VII-3 & Beach sand & $0 \cdot 8$ & 0.5 & $0 \cdot 370$ & $2 \cdot 2 \times 10^{-4}$ & $1 \cdot 040$ & $0 \cdot 241$ & $0 \cdot 241$ \\
\hline $220881-40-1$ & Beach sand & $4 \cdot 5$ & $1 \cdot 5$ & $0 \cdot 360$ & $2 \cdot 2 \times 10^{-4}$ & 1.056 & 0.809 & 0.809 \\
\hline $220881-40-2$ & Beach sand & $4 \cdot 5$ & $1 \cdot 5$ & $0 \cdot 360$ & $2 \cdot 1 \times 10^{-4}$ & $1 \cdot 056$ & 0.715 & 0.715 \\
\hline $220881-40-3$ & Beach sand & $4 \cdot 5$ & $1 \cdot 5$ & $0 \cdot 360$ & $2 \cdot 1 \times 10^{-4}$ & $1 \cdot 056$ & 0.624 & $0 \cdot 624$ \\
\hline $220881-40-4$ & Beach sand & $1 \cdot 2$ & $1 \cdot 5$ & $0 \cdot 360$ & $2 \cdot 1 \times 10^{-4}$ & 1.056 & $0 \cdot 307$ & $0 \cdot 307$ \\
\hline $220881-40-5$ & Beach sand & $1 \cdot 2$ & $1 \cdot 5$ & $0 \cdot 360$ & $2 \cdot 1 \times 10^{-4}$ & 1.056 & $0 \cdot 189$ & $0 \cdot 189$ \\
\hline $220881-40-6$ & Beach sand & $1 \cdot 2$ & $1 \cdot 5$ & $0 \cdot 360$ & $2 \cdot 1 \times 10^{-4}$ & 1.056 & $0 \cdot 288$ & $0 \cdot 288$ \\
\hline $220881-40-7$ & Beach sand & $1 \cdot 2$ & $1 \cdot 5$ & $0 \cdot 360$ & $2 \cdot 1 \times 10^{-4}$ & 1.056 & $0 \cdot 200$ & $0 \cdot 200$ \\
\hline $220883-35-1$ & Coarse sand & $2 \cdot 4$ & $1 \cdot 5$ & $0 \cdot 370$ & $1.8 \times 10^{-3}$ & $1 \cdot 040$ & $0 \cdot 880$ & $0 \cdot 880$ \\
\hline $220883-35-2$ & Coarse sand & $2 \cdot 4$ & $1 \cdot 5$ & $0 \cdot 367$ & $1.5 \times 10^{-3}$ & $1 \cdot 044$ & $0 \cdot 960$ & 0.960 \\
\hline $220883-35-3$ & Coarse sand & $2 \cdot 4$ & $1 \cdot 5$ & $0 \cdot 366$ & $1.5 \times 10^{-3}$ & $1 \cdot 046$ & $0 \cdot 800$ & $0 \cdot 800$ \\
\hline $220883-35-4$ & Coarse sand & $2 \cdot 4$ & $1 \cdot 5$ & $0 \cdot 343$ & $1 \cdot 1 \times 10^{-3}$ & $1 \cdot 084$ & 0.680 & $0 \cdot 680$ \\
\hline $220883-35-5$ & Coarse sand & $2 \cdot 4$ & $1 \cdot 5$ & $0 \cdot 343$ & $1.0 \times 10^{-3}$ & 1.084 & 0.714 & $0 \cdot 714$ \\
\hline $220883-35-6$ & Coarse sand & $2 \cdot 4$ & $1 \cdot 5$ & $0 \cdot 341$ & $1.0 \times 10^{-3}$ & 1.087 & $0 \cdot 885$ & $0 \cdot 885$ \\
\hline $220883-35-7$ & Coarse sand & $2 \cdot 4$ & $1 \cdot 5$ & $0 \cdot 320$ & $8 \cdot 3 \times 10^{-4}$ & $1 \cdot 122$ & 0.626 & 0.626 \\
\hline $220883-35-8$ & Coarse sand & $2 \cdot 4$ & $1 \cdot 5$ & $0 \cdot 320$ & $7 \cdot 5 \times 10^{-4}$ & $1 \cdot 122$ & $1 \cdot 040$ & $1 \cdot 040$ \\
\hline $220883-35-9$ & Coarse sand & $2 \cdot 4$ & $1 \cdot 5$ & $0 \cdot 320$ & $7 \cdot 3 \times 10^{-4}$ & $1 \cdot 122$ & 0.940 & 0.940 \\
\hline $220883-39-1$ & Dune sand & $0 \cdot 8$ & 0.5 & $0 \cdot 390$ & $2 \cdot 6 \times 10^{-4}$ & 1.007 & 0.237 & $0 \cdot 237$ \\
\hline $220883-39-2$ & Dune sand & $0 \cdot 8$ & 0.5 & $0 \cdot 390$ & $2 \cdot 6 \times 10^{-4}$ & $1 \cdot 007$ & $0 \cdot 195$ & $0 \cdot 195$ \\
\hline $220883-39-3$ & Dune sand & $0 \cdot 8$ & $0 \cdot 5$ & $0 \cdot 390$ & $2 \cdot 2 \times 10^{-4}$ & 1.007 & $0 \cdot 214$ & $0 \cdot 214$ \\
\hline 220884-26-1 & Coarse sand & $0 \cdot 8$ & 0.5 & $0 \cdot 369$ & $1.6 \times 10^{-4}$ & $1 \cdot 041$ & $0 \cdot 394$ & $0 \cdot 394$ \\
\hline 220884-26-2 & Coarse sand & $0 \cdot 8$ & $0 \cdot 5$ & $0 \cdot 344$ & $1 \cdot 1 \times 10^{-3}$ & $1 \cdot 082$ & $0 \cdot 391$ & $0 \cdot 391$ \\
\hline $220884-26-3$ & Coarse sand & $0 \cdot 8$ & 0.5 & $0 \cdot 320$ & $8.9 \times 10^{-4}$ & $1 \cdot 122$ & 0.783 & 0.783 \\
\hline $220884-26-4$ & Coarse sand & $0 \cdot 8$ & $0 \cdot 5$ & $0 \cdot 370$ & $1 \cdot 1 \times 10^{-3}$ & $1 \cdot 040$ & 0.792 & 0.792 \\
\hline $220884-26-5$ & Coarse sand & $0 \cdot 8$ & $0 \cdot 5$ & $0 \cdot 345$ & $8.0 \times 10^{-4}$ & $1 \cdot 081$ & 0.660 & $0 \cdot 660$ \\
\hline
\end{tabular}

the head at which sand transport takes place, indicating pipe initiation $\left(H_{\mathrm{i}}\right)$. This corresponds to the findings of Rice \& Swainston-Fleshman (2013), who performed column experiments in which a sand sample was subjected to vertical flow.
They observed sand boils at a lower hydraulic gradient than for total fluidisation in some, but not all, column experiments.

In the small-scale slope-type experiments reported by Van 
Table 3. Additional plane-type experiments

\begin{tabular}{l|l|c|c|c|c|c|c|c}
\hline Test no. & Sand type & $L: \mathrm{m}$ & $D: \mathrm{m}$ & $n$ & $k: \mathrm{m} / \mathrm{s}$ & $i_{\mathrm{c}}$ & $H_{\mathrm{i}}: \mathrm{m}$ & $\mathrm{m}$ \\
\hline I-137 & Enschedé sand & $0 \cdot 33$ & $0 \cdot 1$ & $\begin{array}{l}0 \cdot 322 \\
0 \cdot 323\end{array}$ & $\begin{array}{l}3 \cdot 1 \times 10^{-4} \\
2 \cdot 8 \times 10^{-4}\end{array}$ & $\begin{array}{l}0 \cdot 260 \\
0 \cdot 119\end{array}$ \\
I-138 & Enschedé sand & $0 \cdot 33$ & $0 \cdot 1$ & $0 \cdot 260$ & 0 \\
\hline
\end{tabular}

Table 4. Ditch-type experiments (De Wit, 1984)

\begin{tabular}{|c|c|c|c|c|c|c|c|c|c|c|}
\hline Test no. & Sand type & $L: \mathrm{m}$ & $D: \mathrm{m}$ & $S: \mathrm{m}$ & $n$ & $k: \mathrm{m} / \mathrm{s}$ & $i_{\mathrm{c}}$ & $H_{\mathrm{b}}: \mathrm{m}$ & $H_{\mathrm{i}}: \mathrm{m}$ & $H_{\mathrm{c}}: \mathrm{m}$ \\
\hline $220885-10-1$ & Beach sand & 0.9 & $0 \cdot 5$ & $0 \cdot 05$ & 0.39 & n.a. & $1 \cdot 007$ & $0 \cdot 170$ & $0 \cdot 204$ & $0 \cdot 204$ \\
\hline $220885-10-2$ & Beach sand & $0 \cdot 9$ & 0.5 & $0 \cdot 05$ & $0 \cdot 35$ & n.a. & $1 \cdot 073$ & $0 \cdot 200$ & $0 \cdot 206$ & $0 \cdot 206$ \\
\hline $220885-10-3$ & Beach sand & 0.9 & $0 \cdot 5$ & $0 \cdot 05$ & $0 \cdot 39$ & n.a. & $1 \cdot 007$ & $0 \cdot 144$ & $0 \cdot 144$ & $0 \cdot 144$ \\
\hline $220885-10-4$ & Beach sand & 0.9 & $0 \cdot 5$ & $0 \cdot 05$ & $0 \cdot 35$ & n.a. & $1 \cdot 073$ & $0 \cdot 227$ & $0 \cdot 227$ & $0 \cdot 227$ \\
\hline $220885-10-5$ & Beach sand & 0.9 & $0 \cdot 5$ & $0 \cdot 05$ & $0 \cdot 39$ & n.a. & $1 \cdot 007$ & $0 \cdot 150$ & $0 \cdot 150$ & $0 \cdot 150$ \\
\hline $220885-10-6$ & Beach sand & 0.9 & 0.5 & $0 \cdot 05$ & $0 \cdot 35$ & n.a. & $1 \cdot 073$ & $0 \cdot 225$ & 0.267 & 0.267 \\
\hline $220885-10-6$ & Beach sand & $2 \cdot 7$ & $1 \cdot 5$ & $0 \cdot 05$ & $0 \cdot 35$ & n.a. & $1 \cdot 073$ & $0 \cdot 257$ & $0 \cdot 397$ & 0.397 \\
\hline $220885-10-6$ & Beach sand & $2 \cdot 7$ & $1 \cdot 5$ & $0 \cdot 05$ & $0 \cdot 35$ & n.a. & $1 \cdot 073$ & $0 \cdot 247$ & $0 \cdot 392$ & $0 \cdot 392$ \\
\hline $220885-10-6$ & Beach sand & $2 \cdot 7$ & $1 \cdot 5$ & $0 \cdot 05$ & $0 \cdot 35$ & n.a. & $1 \cdot 073$ & $0 \cdot 222$ & 0.332 & 0.332 \\
\hline
\end{tabular}

Beek et al. (2011), the pipe also developed in the upstream direction after initiation. In the medium-scale experiments, however, some erosion phenomena (pipe formation) were observed at a head that was lower than the critical head. These erosion phenomena involved the formation of pipes that did not start entirely at the downstream end. The initiation head that was selected was the head at which the first pipe formed.

The supplementary plane-type experiments were performed in the small-scale set-up described in Van Beek et al. (2011), but with a plane-type exit instead of a slope-type exit. The critical head in this experiment was found to be dominated by initiation as well, and so $H_{\mathrm{i}}$ equalled $H_{\mathrm{c}}$.

Experiments by Pietrus (1981) and Townsend et al. (1988) were performed in a slope-type configuration with an artificially created pipe. Richards \& Reddy (2012) used a triaxial set-up with a circular pipe as the exit. These experiments have not yet been included in this analysis of initiation because of the $3 \mathrm{D}$ exit configuration.

Tables 2-4 show all the plane- and ditch-type experiments used for the analysis. Van Beek et al. (2011) presented an overview of slope-type experiments but they are presented again here with an improved analysis of the flow resistance of the upstream geotextile filter. Additional experiments looking at low-density sand samples (Van Beek et al., 2012) have also been included. Table 5 shows the successful backward erosion experiments for which the required parameters for analysis of this type of experiment, such as friction angle and slope angle, are known.

\section{INITIATION OF PIPING - THEORY}

On the basis of experiments and theory, Terzaghi (1922) already noted that the magnitude and distribution of seepage forces near the toe of a water-retaining structure, and particularly the discharge gradient at the toe, determine whether piping or underground erosion will occur. The onset of particle movement requires the uplift of particles. The critical heave gradient $\left(i_{\mathrm{c}}\right)$ that causes the fluidisation of the granular material is based on the vertical equilibrium of the granular material, which depends in turn on soil porosity $(n)$, particle density $\left(\rho_{\mathrm{s}}\right)$ and water density $\left(\rho_{\mathrm{w}}\right)$

$$
i_{\mathrm{c}}=-(1-n)\left(\frac{\rho_{\mathrm{s}}-\rho_{\mathrm{w}}}{\rho_{\mathrm{w}}}\right)
$$

This criterion is considered to be valid for internally stable soils that move 'en masse' in the vertical direction as a result of a uniformly distributed upward flow.

Many column experiments have been performed to study this criterion. Experiments of this kind determine the average gradient across the soil sample in the column at which fluidisation occurs. Rice \& Swainston-Fleshman (2013) observed three stages: movement, boiling and then total heave, noting that in some experiments the boiling phase was not seen. The experimentally observed average gradient for the first movement appeared to be close to the Terzaghi heave criterion, but both the first movement of particles and the boiling of sand resulted in an increase in the final critical heave gradient by approximately a factor of 2 as a result of distortion of the sample, resulting in non-uniform flow. In addition, the roughness of the test-device wall and the angularity of the grains appeared to lead to an increase in the critical heave gradient obtained experimentally. Here, it should be noted that the flow pattern at the micro-scale along the wall of the test set-up differs from the flow in a more realistic situation. However, the influence of soil angularity and 'wall' resistance may be relevant factors requiring further research.

Lower values for the critical heave gradient than those found using equation (1) have been observed in internally unstable suffusive soils (Skempton \& Brogan, 1994) or for sloping surfaces. The erosion of grains on a sloping surface, when subjected to a flow perpendicular to the surface, has been investigated by Van Rhee \& Bezuijen (1992), who compared continuum and particle approaches with experimental results. They concluded that a continuum approach for predicting erosion fits the experimental results for outward flow best. This also conforms with theory: if a single grain is lifted from the bed, the seepage forces on this grain immediately fall and the grain falls back to the surface. To cause erosion, a group of particles needs to be uplifted. It is therefore more likely that erosion depends on the exit gradient exerted on a group of grains rather than the exit velocity exerted on a single grain.

The equation for the critical gradient depends not only on 
Table 5. Slope-type experiments (Van Beek et al. (2011) improved filter correction) and scaling parameter $\lambda$

\begin{tabular}{|c|c|c|c|c|c|c|c|c|c|c|}
\hline Test no. & Sand type & $L: \mathrm{m}$ & $D: \mathrm{m}$ & $\beta: \operatorname{deg}$ & $n$ & $k: \mathrm{m} / \mathrm{s}$ & $i_{\mathrm{c}}$ & $H_{\mathrm{i}}: \mathrm{m}$ & $H_{\mathrm{c}}: \mathrm{m}$ & $\lambda^{*}: \mathrm{m}$ \\
\hline B19 & Baskarp sand & $0 \cdot 340$ & $0 \cdot 100$ & 28 & $0 \cdot 386$ & $1.5 \times 10^{-4}$ & $0 \cdot 360$ & $0 \cdot 114$ & $0 \cdot 114$ & $1 \cdot 18$ \\
\hline B23 & Baskarp sand & $0 \cdot 338$ & $0 \cdot 100$ & 28 & $0 \cdot 343$ & $5.9 \times 10^{-5}$ & $0 \cdot 441$ & $0 \cdot 193$ & $0 \cdot 193$ & $1 \cdot 31$ \\
\hline B24 & Baskarp sand & $0 \cdot 338$ & $0 \cdot 100$ & 27 & $0 \cdot 344$ & $6.8 \times 10^{-5}$ & $0 \cdot 464$ & $0 \cdot 172$ & $0 \cdot 172$ & $1 \cdot 30$ \\
\hline B28 & Baskarp sand & $0 \cdot 335$ & $0 \cdot 100$ & 22 & 0.421 & $2.7 \times 10^{-4}$ & $0 \cdot 441$ & $0 \cdot 071$ & $0 \cdot 071$ & $1 \cdot 18$ \\
\hline B35 & Baskarp sand & $0 \cdot 335$ & $0 \cdot 100$ & 23 & $0 \cdot 386$ & $1.3 \times 10^{-4}$ & $0 \cdot 479$ & $0 \cdot 135$ & $0 \cdot 135$ & $1 \cdot 28$ \\
\hline B36 & Baskarp sand & $0 \cdot 334$ & $0 \cdot 100$ & 24 & $0 \cdot 388$ & $1 \cdot 1 \times 10^{-4}$ & $0 \cdot 441$ & $0 \cdot 137$ & $0 \cdot 137$ & $1 \cdot 27$ \\
\hline B40 & Baskarp sand & $0 \cdot 332$ & $0 \cdot 100$ & 22 & $0 \cdot 352$ & $5 \cdot 3 \times 10^{-5}$ & $0 \cdot 571$ & $0 \cdot 148$ & $0 \cdot 148$ & $1 \cdot 38$ \\
\hline B41 & Baskarp sand & $0 \cdot 334$ & $0 \cdot 100$ & 23 & $0 \cdot 350$ & $7 \cdot 3 \times 10^{-5}$ & 0.563 & $0 \cdot 153$ & $0 \cdot 153$ & $1 \cdot 35$ \\
\hline B54 & Baskarp sand & $0 \cdot 330$ & $0 \cdot 100$ & 25 & $0 \cdot 367$ & $7 \cdot 4 \times 10^{-5}$ & $0 \cdot 457$ & $0 \cdot 180$ & $0 \cdot 180$ & $1 \cdot 28$ \\
\hline B55 & Baskarp sand & $0 \cdot 325$ & $0 \cdot 100$ & 21 & $0 \cdot 377$ & $8.8 \times 10^{-5}$ & $0 \cdot 548$ & $0 \cdot 141$ & $0 \cdot 141$ & $1 \cdot 31$ \\
\hline B57 & Baskarp sand & $0 \cdot 330$ & $0 \cdot 100$ & 23 & $0 \cdot 372$ & $8.8 \times 10^{-5}$ & $0 \cdot 508$ & $0 \cdot 132$ & $0 \cdot 132$ & $0 \cdot 89$ \\
\hline B58 & Baskarp sand & $0 \cdot 345$ & $0 \cdot 100$ & 21 & $0 \cdot 379$ & $1.0 \times 10^{-4}$ & 0.536 & $0 \cdot 182$ & $0 \cdot 182$ & $1 \cdot 09$ \\
\hline B61 & Baskarp sand & $0 \cdot 345$ & $0 \cdot 100$ & 24 & $0 \cdot 375$ & $9.9 \times 10^{-5}$ & $0 \cdot 467$ & $0 \cdot 114$ & $0 \cdot 114$ & $0 \cdot 51$ \\
\hline B82 & Baskarp sand & $0 \cdot 336$ & $0 \cdot 100$ & 26 & 0.359 & $5.9 \times 10^{-5}$ & 0.459 & $0 \cdot 139$ & $0 \cdot 139$ & $0 \cdot 98$ \\
\hline B83 & Baskarp sand & $0 \cdot 334$ & $0 \cdot 100$ & 24 & $0 \cdot 359$ & $6.0 \times 10^{-5}$ & $0 \cdot 498$ & $0 \cdot 139$ & $0 \cdot 139$ & $1 \cdot 03$ \\
\hline B84 & Baskarp sand & $0 \cdot 334$ & $0 \cdot 100$ & 25 & $0 \cdot 401$ & $9.7 \times 10^{-5}$ & $0 \cdot 388$ & 0.098 & 0.098 & $0 \cdot 91$ \\
\hline B85 & Baskarp sand & $0 \cdot 336$ & $0 \cdot 100$ & 27 & $0 \cdot 401$ & $7 \cdot 7 \times 10^{-5}$ & $0 \cdot 360$ & $0 \cdot 118$ & $0 \cdot 118$ & 0.93 \\
\hline B86 & Baskarp sand & $0 \cdot 336$ & $0 \cdot 100$ & 27 & 0.414 & $1.0 \times 10^{-4}$ & $0 \cdot 333$ & 0.098 & $0 \cdot 098$ & 0.90 \\
\hline B87 & Baskarp sand & $0 \cdot 336$ & $0 \cdot 100$ & 27 & 0.415 & $1.8 \times 10^{-4}$ & $0 \cdot 331$ & $0 \cdot 046$ & $0 \cdot 046$ & 0.75 \\
\hline B101 & Baskarp sand & $0 \cdot 310$ & $0 \cdot 100$ & 19 & $0 \cdot 408$ & $1.0 \times 10^{-4}$ & $0 \cdot 505$ & $0 \cdot 080$ & $0 \cdot 080$ & 0.90 \\
\hline B103 & Baskarp sand & $0 \cdot 320$ & $0 \cdot 100$ & 20 & 0.438 & $1.6 \times 10^{-4}$ & $0 \cdot 406$ & $0 \cdot 080$ & $0 \cdot 080$ & $0 \cdot 90$ \\
\hline B105 & Baskarp sand & $0 \cdot 335$ & $0 \cdot 100$ & 28 & $0 \cdot 362$ & $7 \cdot 6 \times 10^{-5}$ & $0 \cdot 408$ & $0 \cdot 160$ & $0 \cdot 160$ & 0.94 \\
\hline B107 & Baskarp sand & $0 \cdot 333$ & $0 \cdot 100$ & 28 & $0 \cdot 355$ & $6 \cdot 1 \times 10^{-5}$ & $0 \cdot 421$ & $0 \cdot 180$ & $0 \cdot 180$ & $0 \cdot 95$ \\
\hline B121 & Baskarp sand & $0 \cdot 335$ & $0 \cdot 100$ & 22 & 0.426 & $1.8 \times 10^{-4}$ & $0 \cdot 379$ & $0 \cdot 090$ & 0.090 & $0 \cdot 71$ \\
\hline B122 & Baskarp sand & $0 \cdot 335$ & $0 \cdot 100$ & 21 & 0.430 & $1.6 \times 10^{-4}$ & $0 \cdot 387$ & $0 \cdot 080$ & $0 \cdot 080$ & $0 \cdot 72$ \\
\hline Bms1 & Baskarp sand & $1 \cdot 370$ & $0 \cdot 400$ & 25 & $0 \cdot 392$ & $1 \cdot 2 \times 10^{-4}$ & $0 \cdot 446$ & $0 \cdot 220$ & $0 \cdot 280$ & $1 \cdot 47$ \\
\hline Bms2 & Baskarp sand & $1 \cdot 450$ & $0 \cdot 400$ & 25 & 0.405 & $1.4 \times 10^{-4}$ & $0 \cdot 434$ & $0 \cdot 290$ & $0 \cdot 370$ & $1 \cdot 00$ \\
\hline Bms7 & Baskarp sand & $1 \cdot 300$ & $0 \cdot 400$ & 25 & $0 \cdot 386$ & $1.5 \times 10^{-4}$ & $0 \cdot 451$ & $0 \cdot 140$ & $0 \cdot 290$ & $2 \cdot 08$ \\
\hline Bms8 & Baskarp sand & $1 \cdot 330$ & $0 \cdot 400$ & 25 & $0 \cdot 405$ & $2.6 \times 10^{-4}$ & 0.434 & $0 \cdot 190$ & $0 \cdot 190$ & $2 \cdot 13$ \\
\hline
\end{tabular}

* Obtained by fitting the analytical solution to the numerical solution.

the angle of the sloping surface $(\beta)$ but also on the friction angle $(\phi)$

$$
i_{\mathrm{c}}=-(1-n)\left(\frac{\rho_{\mathrm{s}}-\rho_{\mathrm{w}}}{\rho_{\mathrm{w}}}\right) \frac{\sin (\phi-\beta)}{\sin (\phi)}
$$

Equations (1) and (2) will be used in the subsequent analysis in the present paper as the criteria for the initiation of piping.

\section{EXIT GRADIENT ANALYSIS}

Most experiments to investigate heave criteria are performed in columns: test set-ups with a unidirectional flow, with the average gradient in the soil sample $H / L$ equalling the exit gradient (here: $i$ ). In backward erosion experiments, however, the flow pattern is not unidirectional. Large flow differences are present near the structure toe on the downstream side. The average gradient $H / L$ across the structure is therefore not equal to the local exit gradient $i$ near the toe on the downstream side. Given an average gradient across the structure $(H / L)$, the exit gradient $(i)$ can be calculated as a function of the distance from the toe using groundwater calculations for the specific geometry.

To determine the conditions at which a pipe is initiated, it is necessary to determine the relationship between the local exit gradient $(i)$ near the toe of the structure and the overall average gradient $(H / L)$. The exit gradient is largely dependent on the geometry of the outflow surface and on the shape and size of the sand layer. Several types of geometry are considered here - plane-, ditch- and slope-type (Fig. 1) - which correspond to the geometries of experiments in the laboratory.

To calculate the exit gradient, analytical solutions are preferable to numerical solutions because they provide more accurate results close to the exit. Polubarinova-Kochina (1962) provides a solution for the exit gradient $(i)$ at the surface downstream of the toe for a confined aquifer (below a dam) with a plane-type exit. Using this equation (3), the exit gradient $i$ can be calculated for the plane-type experiments (Fig. 1(a)), which, in essence, simulate the downstream-half of the dam described in Polubarinova-Kochina (1962). In equation (3), the $x$-coordinate is defined as the distance from the upstream filter, the thickness of the sample is $D$, the head drop applied over the sand sample is $H$, the shortest distance from the upstream filter to the downstream plane is the seepage length $L$, and $K$ is a complete elliptic integral of the first kind over $m$, as seen in equation (4).

$$
i=\frac{H \pi}{2 K D} \frac{\cosh (\pi L / 2 D)}{\sqrt{\sinh \left[\frac{\pi(L+x)}{2 D}\right] \sinh \left[\frac{\pi(x-L)}{2 D}\right]}}
$$

in which

$$
K=\int_{0}^{\frac{1}{2} \pi} \frac{\mathrm{d} \phi}{\sqrt{1-m \sin ^{2} \phi}} \quad m=\tanh ^{2}\left(\frac{\pi}{2} \frac{L}{D}\right)
$$

There are no standard solutions available for the flow in a ditch-type configuration. Using conformal mapping, the complex potential $\Omega(\Omega=\Phi+i \Psi=k \varphi+i \Psi$, in which $\Phi$ is the groundwater potential, $k$ the permeability, $\varphi$ the groundwater head and $\Psi$ the stream function as a function of the complex coordinate $z(z=x+i y))$ can be obtained for this configuration (Fig. 2). The geometry in the $z$-plane can be mapped onto the $\Omega$-plane, where boundary $\mathrm{AB}$ is a line of constant potential (equal to 0 ) and line $\mathrm{CC}$ with a constant potential is situated at infinity on the right. Line DD is situated at infinity at the left and has no prescribed 

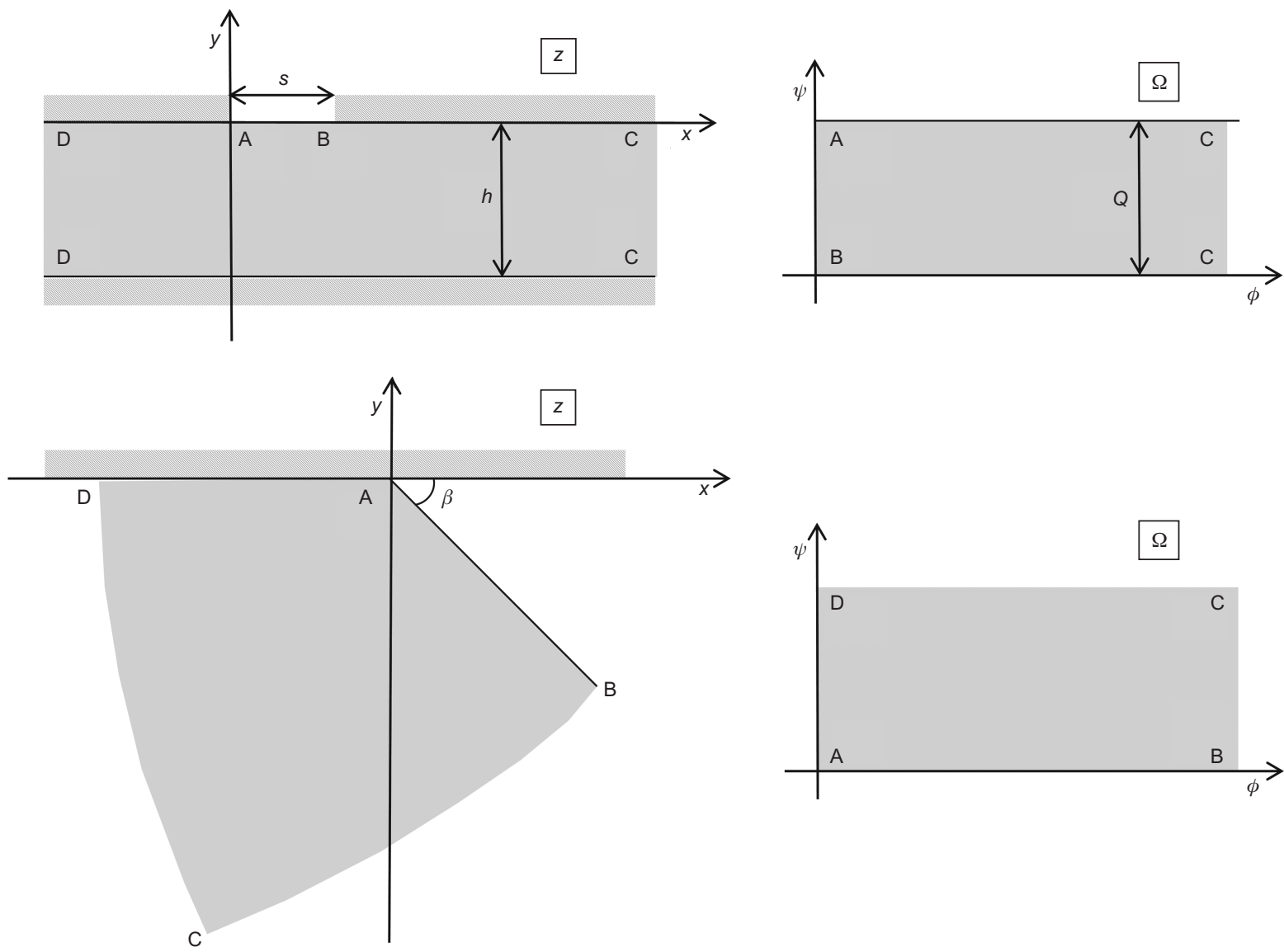

Fig. 2. Conformal mapping schemes ditch (top) and slope (bottom)

potential. The entire flow passes $\mathrm{AB}$ and $\mathrm{CC}$, and it is equal to $Q$. Equation (5) fulfils this mapping. The sample thickness is defined as $h$ and the width of the ditch is $s$ (Fig. 2).

$$
\cosh ^{2}\left(\frac{\pi}{2} \frac{\Omega}{Q}\right)=\frac{\exp [\pi(z / h)]-1}{\exp [\pi(s / h)]-1}
$$

The complex velocity

$$
\bar{w}=-k \frac{\partial \phi}{\partial x}+k \mathrm{i} \frac{\partial \phi}{\partial y}
$$

is obtained by taking the derivative of equation (5) with respect to $z$

$$
\begin{aligned}
& -\frac{\bar{w} h}{Q} \cosh \left(\frac{\pi}{2} \frac{\Omega}{Q}\right) \sinh \left(\frac{\pi}{2} \frac{\Omega}{Q}\right)=\frac{\exp [\pi(z / h)]}{\exp [\pi(s / h)]-1} \\
& \frac{\mathrm{d} \Omega}{\mathrm{d} z}=-\bar{w}
\end{aligned}
$$

Rewriting and substituting the boundary condition for $\mathrm{AB}$ (complex component of $z$ equals 0 ) results in equation (7), which gives the complex velocity for line $\mathrm{AB}$.

$$
\bar{w}=\frac{1}{h} \frac{-Q}{\sqrt{1-\exp \left(\pi \frac{-x}{h}\right)} \sqrt{1-\exp \left(\pi \frac{s-x}{h}\right)}}
$$

in which $Q$ can be defined using equation (5) by filling in the upstream potential $k \varphi_{0}$ at distance $L+s$, assuming that this distance is large enough to make $Q$ constant $\cosh ^{2}\left(\frac{\pi}{2} \frac{k \phi_{0}}{Q}\right)=\frac{\exp \left(\pi \frac{L+s}{h}\right)-1}{\exp [\pi(s / h)]-1}$

The exit gradient is then obtained by dividing the modulus of the velocity $w$ by the permeability $k$

$$
\begin{aligned}
i=\frac{|\bar{w}|}{k}= & \frac{\pi H}{2 h}\left\{\sqrt{1-\exp \left(\pi \frac{-x}{h}\right)} \sqrt{1-\exp \left(\pi \frac{s-x}{h}\right)}\right. \\
& \left.\times \operatorname{arccosh} \sqrt{\frac{\exp \left(\pi \frac{L+s}{h}\right)-1}{\exp [\pi(s / h)]-1}}\right\}^{-1}
\end{aligned}
$$

For larger values of $s$ the gradient $i$ obtained with equation (9) converges to the value for $i$ obtained with equation (3) for plane-type experiments.

The exit gradient $i$ in the slope-type experiments is not determined analytically since the semi-permeable downstream filter makes analytical analysis impossible. It should be noted that these experiments were not designed for initiation analysis. Given this, a numerical calculation was conducted using the two-dimensional (2D) finite-element model MSeep (GeoDelft, 2002). Numerical models can be refined to deliver output very near to the singularity, but this will always result in an averaged value for the exit gradient rather than an exact value. The numerical analysis is therefore combined with an exact solution that is likely to be valid near the singularity.

The exact solution for groundwater flow near a sloping exit is determined using conformal analysis. The conformal 
mapping scheme for this situation is shown in Fig. 2 (lower figures).

The complex potential as a function of a complex coordinate is

$$
\frac{\Omega}{k H}=\left(-\frac{z}{\lambda}\right)^{1 /(2 \alpha)} \quad \alpha=1-\frac{\beta}{\pi}
$$

in which $\lambda$ represents a scaling parameter and $\beta$ is the slope angle with the horizontal. The exit velocity can be obtained by taking the derivative of the complex potential to $z$.

$$
\begin{aligned}
\frac{\mathrm{d} \Omega}{\mathrm{d} z} & =-\bar{w} \\
\bar{w} & =\frac{k H}{2 \alpha \lambda}\left(-\frac{z}{\lambda}\right)^{[1 /(2 \alpha)]-1}
\end{aligned}
$$

The absolute value of the exit velocity $w$ is now expressed as a function of the distance along the slope, $r$, by replacing $z\left(z=r \mathrm{e}^{-i \beta \pi}\right)$. As the modulus of the exponential of any imaginary number is equal to one, the absolute value of the exit gradient can be written as in equation (12), after dividing the modulus of the complex exit velocity by the permeability.

$$
i=\frac{|\bar{w}|}{k}=\frac{H}{2 \alpha \lambda}\left(\frac{r}{\lambda}\right)^{[1 /(2 \alpha)]-1}
$$

Since $\lambda$ is a scale parameter, it is easy to conclude from equation (12) that the effect of scale (denoted by $L$ or $\lambda$ ) on the average gradient $(H / L)$ is defined by the inverse square root of $L$ if $\alpha=1$, thus $\beta=0$ (see equation (10)). As will be shown later, this effect is equal to the effect found in plane-type experiments.

Figure 3 shows an example of the flow net and exit gradient profile obtained with equations (10) and (12). Fitting the scaling parameter $\lambda$ to the numerical results gives the exit gradient profile near the singularity (Fig. 3, right). It should be noted that the analytical solution is only valid when the sample can be regarded as a continuum.

\section{EXPERIMENT ANALYSIS}

The exit gradient analysis in the previous section allows for the calculation of the actual exit gradient in the experiments at the head at which a pipe was initiated.

Heave can only occur if a group of grains is subjected to a certain gradient. However, theoretically, the exit gradient increases to infinity when the toe of the embankment, which is a singular point, is approached. In reality, the flow will be controlled by the micro-scale processes around the grains. In this approach it is assumed that the gradient across a group of grains will determine whether heave will take place. The actual size of this group is unknown. However, the size of the expansion area has been observed in experiments and it is estimated to be $15-20 \mathrm{~mm}$ wide for fine sands (De Wit, 1984), which may be an indication of the distance across which the sand needs to be fluidised. The present study verified whether the initiation gradient $H_{\mathrm{i}} / L$ was indeed determined by local fluidisation near the toe of the simulated embankment. The equations in the previous section - which describe the relationship between exit gradient and head across the structure - were used for this purpose. With the initiation gradients $H_{\mathrm{i}} / L$ obtained from the experiments being applied, the exit gradients $(i)$ were calculated as a function of distance from the toe for the situation in which the pipe was initiated. Subsequently, the distance $d x$ from the embankment toe was calculated at which the actual exit gradient $i$ in the experiments equalled the critical heave gradient $i_{\mathrm{c}}$ (this concept is illustrated in Fig. 4). This distance can be expressed as a number of grains in the lateral direction and will be denoted as the group size $a$.

It is argued here that the size of the group of grains that has to be uplifted to initiate a pipe is independent of the scale and configuration of the set-up and grain size. To verify this, the size of the group of grains to be uplifted is calculated in the following sections using the initiation gradients obtained from the available experiments. If the hypothesis is true, the calculated group size should be in the same range for all experiments, without variations depending on scale or grain size.

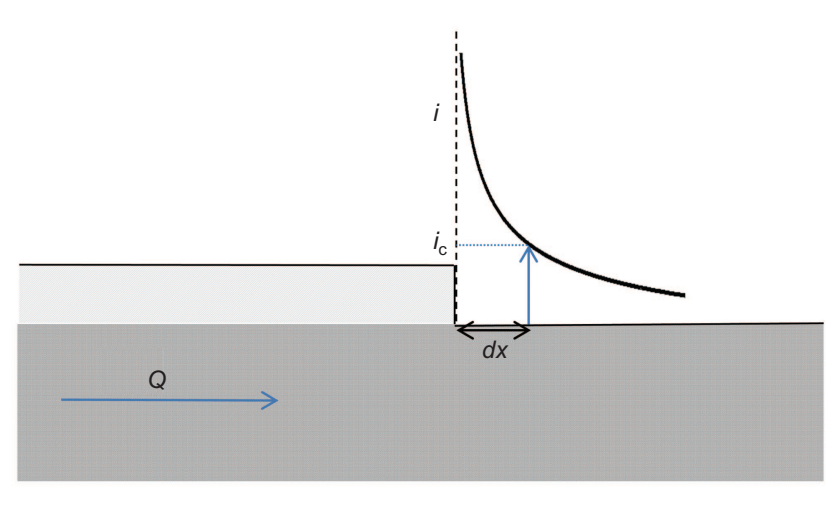

Fig. 4. Conceptual model to determine the group size
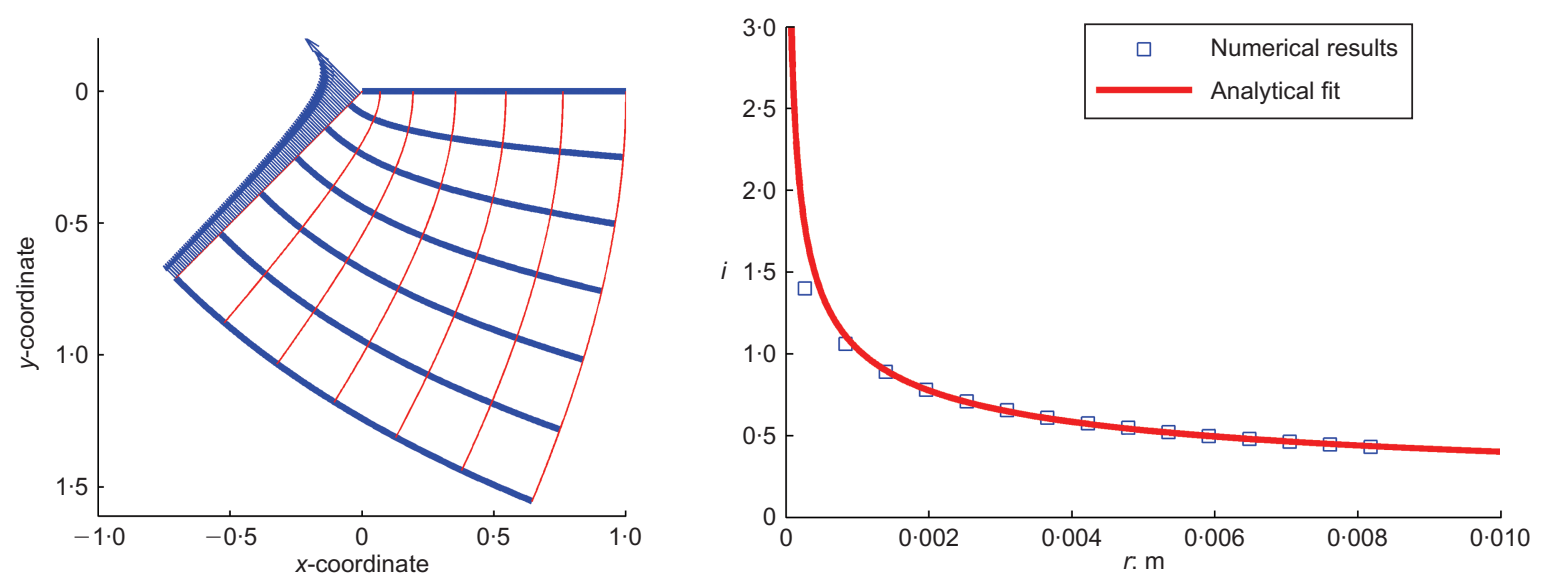

Fig. 3. Flow net and corresponding numerically obtained exit gradients and analytical fit for experiment B19 
Plane-type experiments

The theoretical critical heave gradient $\left(i_{\mathrm{c}}\right)$ was calculated for all available plane-type experiments (displayed in Tables 2 and 3) using equation (1). Using the geometry of the sand layer and the initiation gradient as observed in the experiments, the actual exit gradient profile along the surface was calculated as a function of the distance from the toe. The distance $d x$ from the simulated embankment toe at which the critical heave gradient $i_{\mathrm{c}}$ equalled the exit gradient $i$ was calculated for each experiment.

Using this approach, it is possible to explain scale effects, that is to say differences in initiation gradients $H_{\mathrm{i}} / L$ as a result of differences in the dimensions of the set-up (also reported in Van Beek et al. (2013)). The obtained distances $d x$, which are an indication of the size of the group to be uplifted, were calculated for all available plane-type experiments with Beach sand to observe the influence of scale. The distance $d x$ is expressed as the number of grains $a$ in the lateral direction by

$$
d x=a d_{50}
$$

Figure 5 (left) shows that calculations of the typical group size $a$ in Beach sand experiments result in values that are approximately 20-60 times the average grain diameter (or 4-12 $\mathrm{mm}$ ), regardless of the dimensions of the experiment. This number is in the same order of magnitude as the size of the expansion area observed in these experiments. The large scatter in results is, in part, linked to experimental deviations, as can be observed from the experiments with $L=1.2 \mathrm{~m}$; these experiments were identical in all aspects but there was considerable scatter in the outcomes. Given the proposed theory that local fluidisation of the sand bed in a very small zone initiates piping, this seems reasonable. The experimental scatter in the initiation gradient $H_{\mathrm{i}} / L$ may

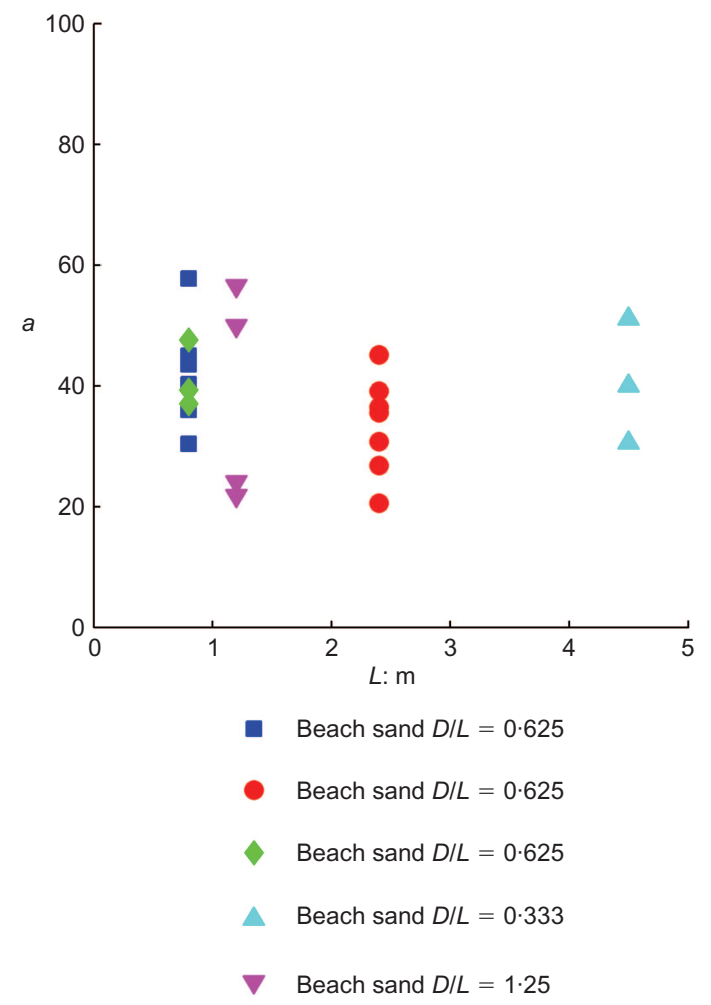

have been caused by small variations in density and permeability that have a large impact on the exit gradient near the toe of the simulated embankment. There is uncertainty about the homogeneity of the compaction and the degree of saturation for the experiments of De Wit (1984), and this may explain the scatter.

Although the analysis included experiments with different dimensions ( $L$ and $D / L)$ and consequently variation in the initiation gradients $H_{\mathrm{i}} / L$, the group size $a$ was not found to be linked to these parameters. This analysis therefore shows that differences in initiation gradient $\left(H_{\mathrm{i}} / L\right)$ as a result of the scale and shape of the sand sample can be explained by the relationship between the gradient across the structure $H / L$ and exit gradient $i$ exerted on a small group of grains. The scale effects can be explained as follows: the linearity of the laminar groundwater flow causes the flow velocity distribution to be linearly dependent on scale, but the size of the grains does not change with scale. Consequently, when considering the exit gradient for a group of grains in a largescale experiment, this group of grains is closer to the toe of the embankment in relative terms than in a small-scale experiment. As the convergence of flow lines near the toe of the structure causes the exit gradient $i$ there to rise (theoretically) to infinity (Fig. 4), the exit gradient across this group is higher when the group of grains is situated closer to the structure toe. This is seen in Fig. 4, which shows a group size $d x$ as well as the corresponding exit gradient $i$. As the curve of the exit gradient in Fig. 4 is independent of scale, a larger scale can be imagined in which the group size $d x$, which does not change with scale, is situated closer to the toe in relative terms and is therefore subject to a higher exit gradient $i$.

In large-scale experiments, therefore, the initiation gradient $H_{\mathrm{i}} / L$ is relatively low, since the group of grains to be

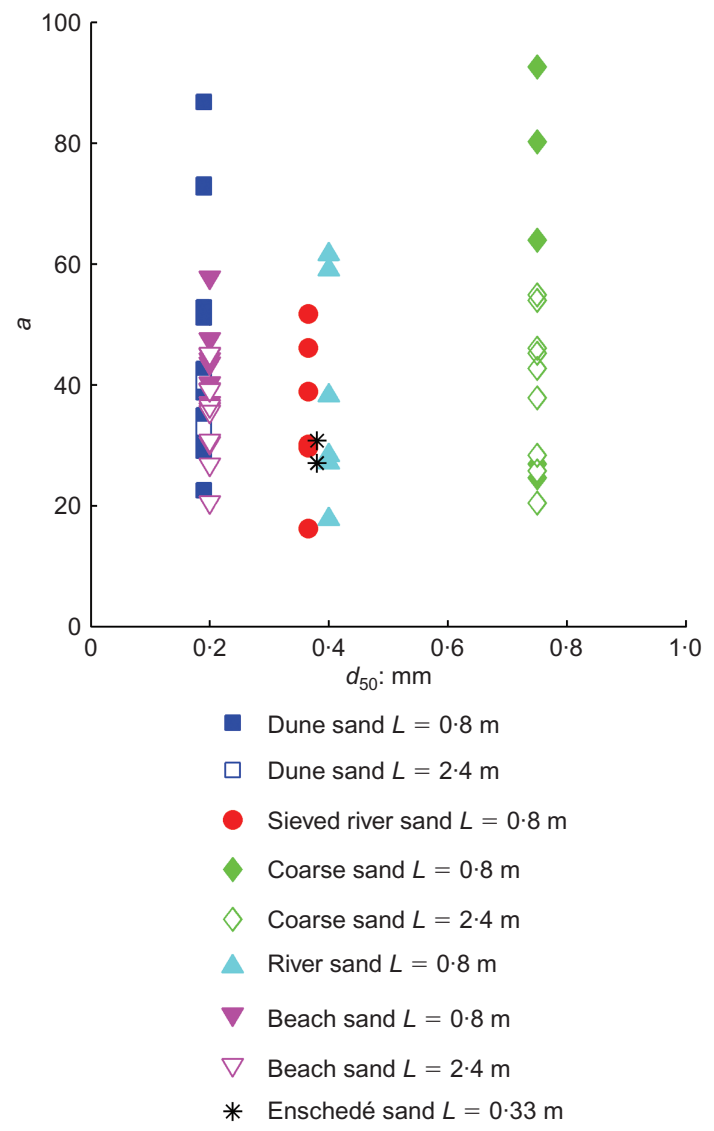

Fig. 5. Calculated heave group size as function of seepage length and grain size (experiments by De Wit (1984) and additional small-scale experiments on Enschedé sand) 
fluidised is located close to the toe of the embankment in relative terms (and thus close to the singularity) by comparison with the overall dimensions of the set-up. In small-scale experiments the initiation gradient $H_{\mathrm{i}} / L$ is relatively high as the group of grains is located further from the toe in relative terms.

The effect of grain size on the initiation gradient (Fig. 5, right) can be analysed in a similar manner. If it is assumed that the size of the group of grains that will be uplifted, expressed as a number of grains, remains the same, the experiment with larger grains requires the exit gradient $i$ to equal the critical heave gradient $i_{\mathrm{c}}$ at a larger distance from the toe, and this results in a higher overall gradient across the sand sample.

Figure 5 (right), which shows the calculated group size for different sand types, validates the explanations given here. Fig. 5 shows that the group of grains to be transported is independent of grain size or scale, and even shape (varying $D / L$ ratio). The minimum group size appears to be approximately 20 grains. Until now, it has been assumed that a continuum approach is valid. Al Hattamleh et al. (2009) prove that the representative elementary volume (REV) radius for estimating the porosity of rounded sand is approximately 9-16 times the $d_{50}$. It can be expected that the REV radius for groundwater flow will be in the same range. The continuum approach is therefore likely to be valid for a range of 20 grains.

The influence of porosity on the critical head was investigated for several sand types. Fig. 6 (left) shows that the relationship between porosity and initiation gradient is not always clear due to the scatter of the initiation gradients obtained in experiments. A clear trend was obtained in two experiment series - the experiments on Beach sand and Dune sand - with the main difference being the friction angle. The heave group size $a$ for these experiments is presented in Fig. 6 (right).

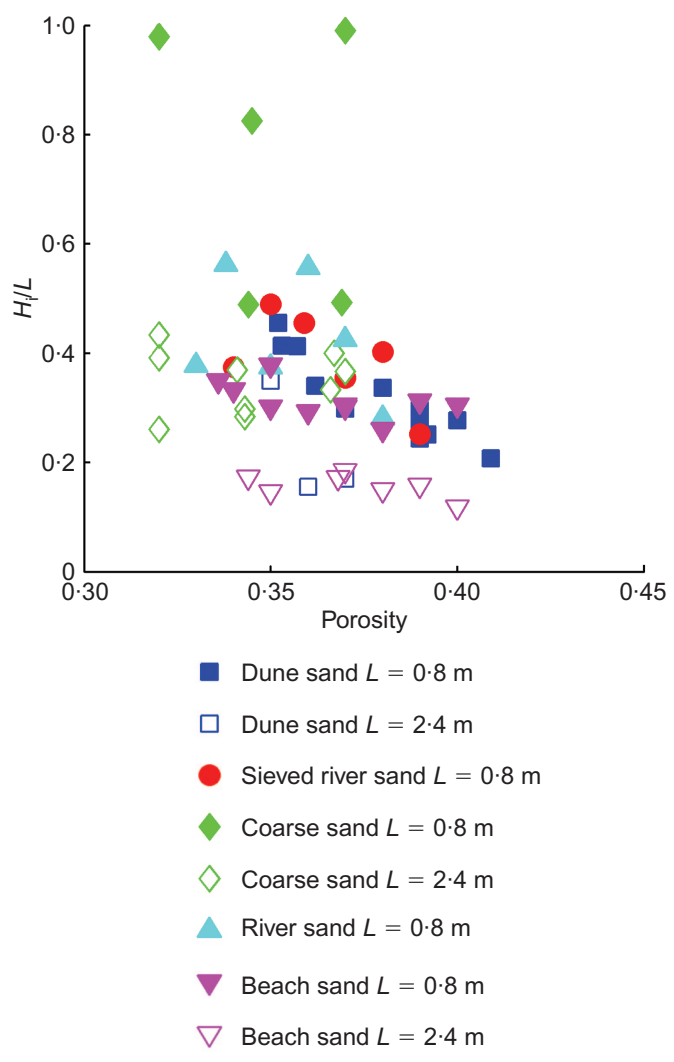

It can be seen that the trend observed in Fig. 6 - that is to say the fall in the initiation gradient with increasing porosity - is not fully corrected by a change in the critical heave gradient $i_{\mathrm{c}}$ alone. The group size should be constant when the effect of porosity has been included correctly but, particularly in the case of Dune sand, a fall in group size was observed as porosity increased. Large group sizes found in experiments on sand with low porosity indicate that the initiation gradient is larger than in the high-porosity samples. This behaviour could be explained by the inter-particle friction, which is not taken into account in the current approach. Rice \& Swainston-Fleshman (2013) report that wall friction has a significant effect on the critical heave gradient: silicon-coated columns result in critical gradients that are approximately 1.5 times higher than in the case of smooth columns. Loezos et al. (2002) performed gas fluidisation in sand columns. They noted that wall friction is the main cause of pressure overshoot and that it increases with decreasing diameter of the column. In backward erosion experiments, where only part of the sample fluidises, the 'wall' consists of those sand particles that are not fluidised. Given that the area of fluidisation is very small, the friction at the interface of fluidised and non-fluidised sand could affect the fluidisation gradient. The 'wall friction' for fluidisation of a small local zone in a larger sand sample is therefore likely to depend on the friction angle of the sand and the interlocking of particles. The friction angles for Dune sand are higher than those for Beach sand. The difference in friction angle could be related to the grain roundness. A comparison of the particles of both sand types with grain roundness classification charts showed that Dune sand is slightly more angular than Beach sand. Another possible explanation for the difference in initiation gradients for the two sand types is the distortion of the sand sample where it has expanded and loosened as a result of water

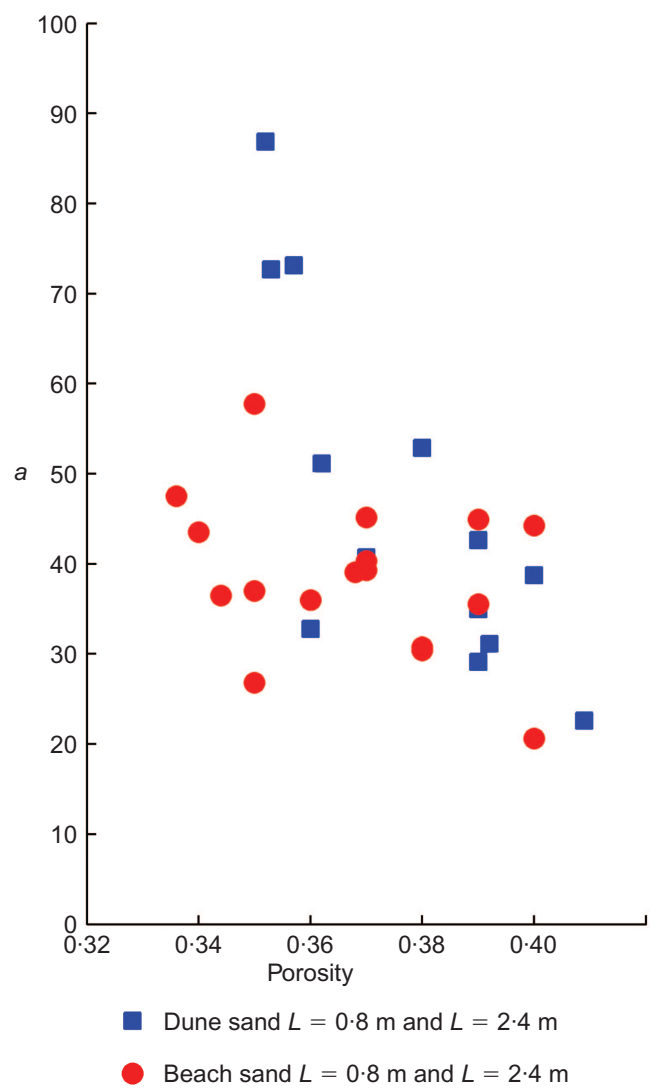

Fig. 6. Influence of porosity on critical gradient and calculated heave group size as a function of porosity for Dune sand and Beach sand experiments 
pressure, a process that takes place in dense sand samples before the sand is fluidised. If the sand is loosened near the toe only, the total flow towards the exit will not increase. The increase of permeability in this loose zone near the toe will therefore result in a local fall in the exit gradient. As a result, the initiation gradient $H_{\mathrm{i}} / L$ needs to be increased before total fluidisation can occur near the toe. Although this may explain why group size $a$ varies with porosity, it cannot explain the difference in the trend for porosity and group size for Beach sand and Dune sand since the increase in permeability with porosity (which can be derived from the relationship between porosity and permeability using the values in Table 2) is comparable for both types of sand. The cause of the difference between the trends therefore remains rather unclear. Experimental scatter or the difference in wall friction, caused by differences in the friction angle or the interlocking of grains, remain as speculative explanations.

\section{Ditch-type experiments}

The available ditch-type experiments are listed in Table 4. The influence of scale and porosity on the critical head have been investigated using Beach sand (De Wit, 1984). Using equation (9), the exit gradient $i$ can be calculated at the surface of the ditch. Using the same approach described above (under Plane-type experiments) for each experiment, the distance $d x$ was determined at which the exit gradient $i$ equalled the critical heave gradient $i_{\mathrm{c}}$ calculated with equation (1).

In some experiments, it was found that the calculated exit gradient $i$ exceeded the heave gradient $i_{\mathrm{c}}$ at every location in the ditch. In these experiments (mainly the larger-scale experiments and experiments with dense sand only), the difference between the required head for sand boiling $H_{\mathrm{b}}$ and sand transport $H_{\mathrm{i}}$ was large. When applying the head for sand boiling $H_{\mathrm{b}}$ instead of the head at which the first sand transport is observed $H_{\mathrm{i}}$, the exit gradient was found to be slightly smaller across the width of the ditch, making it possible to determine the heave group size in most of the experiments.

Van Beek et al. (2013) described the influence of the configuration of the set-up on the initiation and progression heads. Large-scale experiments and experiments with small exit areas are likely to be dominated by progression rather than by initiation. It is likely that the small exit area in these experiments resulted in a reduction of the sand boiling head. As the ditch width was the same in all ditch-type experiments, the exit area was small in relative terms in the large-scale experiments. It is possible that, although the observations in the experiments match the observations in plane-type experiments (De Wit, 1984), the required head for progression exceeded the head for pipe initiation, causing the critical head to be higher than might have been expected on the basis of initiation theory. Another possible explanation of the large initiation gradients in ditch-type experiments could be that the grains are locked in more tightly in the ditch-type experiments than in the plane-type experiments because of the concentration of flow lines towards the ditch (bridging). A third explanation is that the dense sand samples result in higher wall friction, a result of inter-particle friction and the interlocking of grains, leading to more resistance than would be expected on the basis of the critical heave gradient. Finally, the distortion of the sample due to loosening before fluidisation takes place is likely to reduce the exit gradient, as was the case in the plane-type experiments. However, expansion is likely to occur in a larger area in a ditch-type experiment since the exit gradient is also high for the downstream side of the ditch, and so the effect on the exit gradient $i$ differs from that in the plane-type experiments.
Figure 7 shows the group sizes obtained using the sand boiling head $H_{\mathrm{b}}$. Although only a few experiments are available, it is reasonable to conclude that the group sizes in experiments with loose sand are in the same range as with area-type experiments, but that there is more scatter. The calculated group size appears to be in the same range at different scales, suggesting that scale effects can be explained satisfactorily using this theory.

\section{Slope-type experiments}

Slope-type experiments were performed for different sand types, porosities and scales (small $L \cong 0.35 \mathrm{~m}$ and medium $L \cong 1.40 \mathrm{~m}$ ). The experiments were not designed to study the initiation of piping. Furthermore, relevant parameters such as the slope angle and geometry were not determined accurately during the experiments. Nevertheless, the experiments were dominated by the process of initiation, and pipe formation was seen to occur where the exit gradients were highest (Fig. 3) near the top of the sand sample, as in the plane- or ditch-type experiments.

Table 5 lists the experiments for which relevant parameters are available. The critical heave gradient $i_{\mathrm{c}}$ was calculated using equation (2). The slope angle, which was determined using photographs of the experiments, has a major impact on the critical heave gradient $i_{\mathrm{c}}$ and this resulted in a wide margin of inaccuracy.

Figure 8 shows the slope angle determined as a function of porosity for Baskarp sand. A very weak correlation was observed between slope angle and porosity, but there was considerable scatter. A correlation was expected as the slope was formed naturally after the removal of part of the sand sample. Theoretically, the slope angle should equal the angle of repose, which is related to the porosity. The scatter in slope angles was presumably caused by the difficulty of determining this angle using photographs. In the mediumscale experiments, the slope angle could not be determined using the pictures. The slope angle was estimated on the basis of the slope angles observed in the small-scale experiments using a least-square correlation with porosity. The slope angle variation for dense sand samples $\left(22-28^{\circ}\right)$ results in a range for the critical heave gradient $\left(i_{\mathrm{c}}\right)$ of $0.58-0.42$, which indicates the degree of inaccuracy that can also be expected when predicting the initiation gradient for this type of experiment.

Using the initiation gradients in Table 5, and the numerical and analytical analysis of the exit gradient, the group

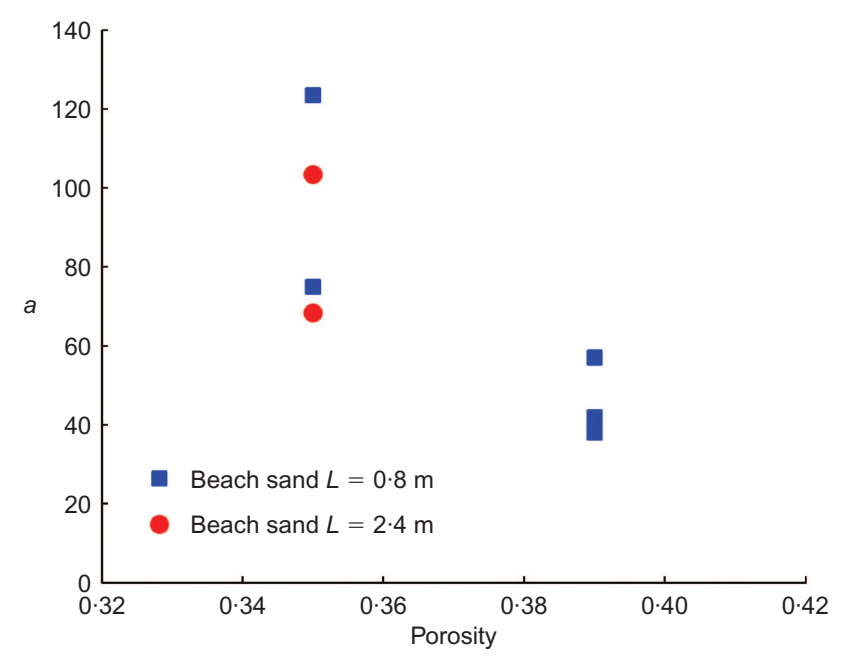

Fig. 7. Calculated heave group size as function of porosity for Dune sand experiments with ditch-type exit 


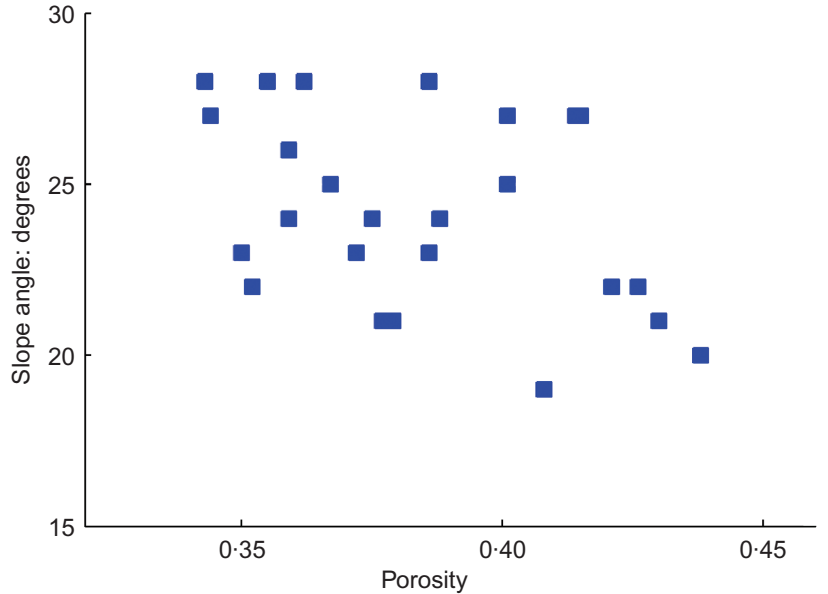

Fig. 8. Observed slope angles as a function of porosity for smallscale Baskarp experiments

size $a$ was determined for each experiment. Fig. 9 shows the group sizes obtained as a function of porosity. The scatter in experiments with this configuration was larger than in experiments with plane- and ditch-type configurations. Local variations in density or poor saturation are unlikely to have caused the scatter since the slope-type experiments were prepared using the method proposed by Poel \& van der Schenkeveld (1998), which ensures a well-saturated and homogeneous sample. The relatively short time interval used for the incremental steps in the head increase in the slopetype experiments could be another explanation for the scatter, but this is also considered to be unlikely. Although, in other types of internal erosion experiments, the time interval matters because the detachment of particles takes time and the gradual detachment of fines will alter the sample, the role of fine grain detachment will be negligible with the internally stable and uniform sands considered in the present paper. The process of backward erosion relies on

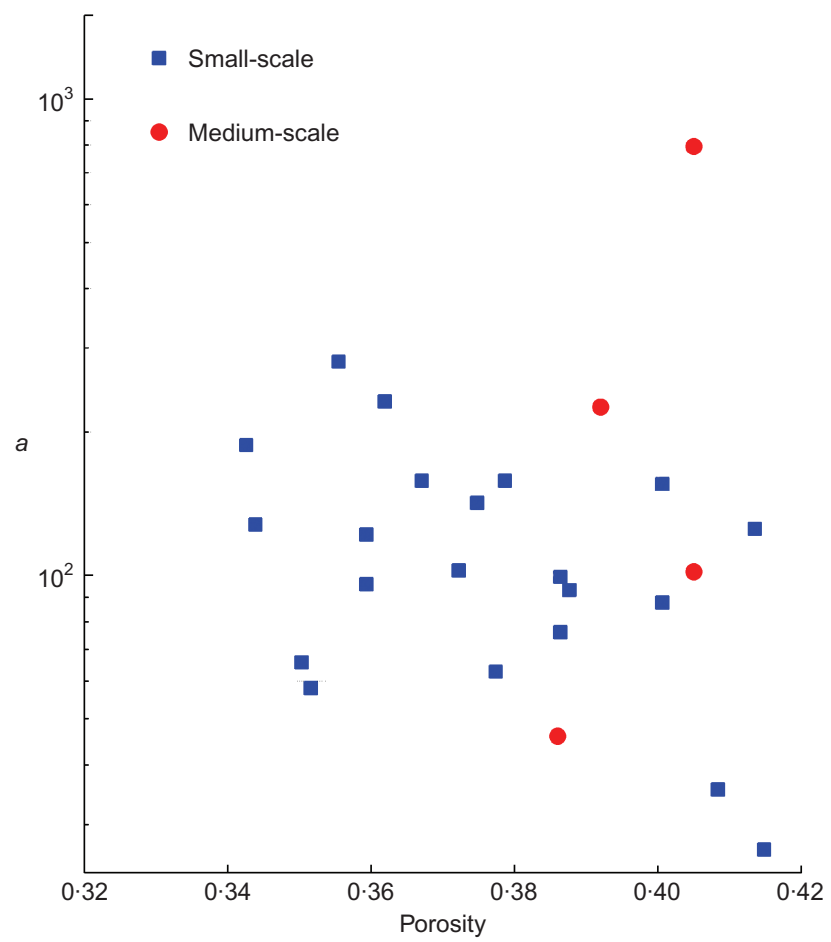

Fig. 9. Calculated group sizes for small- and medium-scale slopetype experiments the removal of groups of sand grains rather than the removal of fines from a matrix. The most likely causes of the scatter are therefore the variation in the slope angle, which was difficult to measure using photographs, or random irregularities at the interface of the sand and the acrylate cover.

Most of the medium-scale results are covered by the scatter for the small-scale results, although the initiation gradients $\left(H_{\mathrm{i}} / L\right)$ in medium-scale experiments are much lower than those in small-scale experiments. This indicates that the scale effect can be explained by the relationship between exit gradient $i$ and $H$, as is stated in equation (12). The relationship between group size and porosity is similar to the relationships observed in the other configurations.

The range of group sizes obtained is not very different from group sizes obtained for the other configurations, which ranges from 24 to 232 in the small-scale experiments. In the Dune sand area-type experiments, the group size is still dependent on porosity, which could be explained by the inter-particle friction and interlocking of grains, resulting in high wall friction, or by the loosening of the sand bed as a result of increasing water pressures. Despite the large scatter and the unintended use of the experiments for pipe initiation analysis, the experiments seem to confirm the theory.

\section{DISCUSSION}

Equations for the relationship between the overall gradient $(H / L)$ and the exit gradient $(i)$ on the one hand and the experimental initiation gradients $\left(H_{\mathrm{i}} / L\right)$ on the other have been used here to determine the group size $a$ across which the sand needs to be fluidised to cause pipe initiation. A minimum group size of approximately 20 grains was calculated for all experiments. This empirically obtained value allows for the prediction of a minimum value for the initiation head $H_{\mathrm{i}}$ across the structure that causes pipe initiation. Using the equations proposed in the earlier section on 'Exit gradient analysis', in which the head drop across the structure $H$ is related to the exit gradient $i$ in different configurations, the initiation gradient $H_{\mathrm{i}} / L$ can be calculated as the head at which the exit gradient $i$ equals the heave gradient $i_{\mathrm{c}}$ at a distance from the toe of 20 times the mean diameter of the sand $\left(x=20 d_{50}\right)$.

Table 6 shows the equations for the configurations with a plane-type exit and a ditch-type exit that predict the initiation gradient $H_{\mathrm{i}} / L$. In the case of the slope-type exit, a numerical calculation is required to determine the parameter $\lambda$ and therefore the equation is not presented here. However, once this parameter has been determined, the initiation gradient $H_{\mathrm{i}} / L$ can be determined in the same way by calculating the gradient $H / L$ at which the exit gradient $i$ equals the critical heave gradient $i_{\mathrm{c}}$ for a distance along the slope of 20 times the mean grain diameter $\left(r=|z|=20 d_{50}\right)$.

The equations show that the scale (variation of $L$ for constant $D / L$ ) and grain size affect the initiation gradient $H_{\mathrm{i}} / L$. The initiation gradient was plotted as a function of the seepage length for the plane- and ditch-type configuration while all other parameters were kept constant (Fig. 10). It emerged that, in both configurations, the initiation gradient changes in line with the inverse of the square root of $L\left(H_{\mathrm{i}} / L \propto 1 / \sqrt{L}\right)$. Bezuijen \& Steedman (2010) already derived this scaling factor theoretically for a configuration with infinite depth.

Using the equations in Table 6 and the combined numerical/ analytical approach described in the earlier section on 'Exit gradient analysis' for the slope experiments, the initiation head $H_{\mathrm{i}}$ was calculated for all experiments evaluated in this paper (Fig. 11). This model provides a good explanation of the differences in the initiation gradient associated with scale, configuration and grain size, as can be seen in Fig. 11.

Figure 11 shows that the approach is conservative. This is 
Table 6. Equations for prediction of initiation gradient for plane- and ditch-type configurations

\begin{tabular}{l|l}
\hline Configuration & Equation \\
\hline Plane-type exit & $\frac{H_{\mathrm{i}}}{L}=i_{\mathrm{c}} \frac{2 K}{\pi} \frac{D}{L} \frac{\sqrt{\sinh \left[\frac{\pi\left(2 L+20 d_{50}\right)}{2 D}\right] \sinh \left[\frac{\pi\left(20 d_{50}\right)}{2 D}\right]}}{\cosh (\pi L / 2 D)}$ \\
in which & $K=\int_{0}^{\frac{1}{2} \pi} \frac{\mathrm{d} \phi}{\sqrt{1-m \sin ^{2} \phi}} \quad m=\tanh ^{2}\left(\frac{\pi}{2} \frac{L}{D}\right)$ \\
Ditch-type exit & $\frac{H_{\mathrm{i}}}{L}=-i_{\mathrm{c}} \frac{2}{\pi} \frac{D}{L} \sqrt{1-\exp \left(\pi \frac{20 d_{50}-s}{D}\right)} \sqrt{1-\exp \left(\pi \frac{20 d_{50}}{D}\right)} \operatorname{arccosh} \sqrt{\frac{\exp \left(\pi \frac{L+s}{D}\right)-1}{\exp \left(\pi \frac{s}{D}\right)-1}}$
\end{tabular}

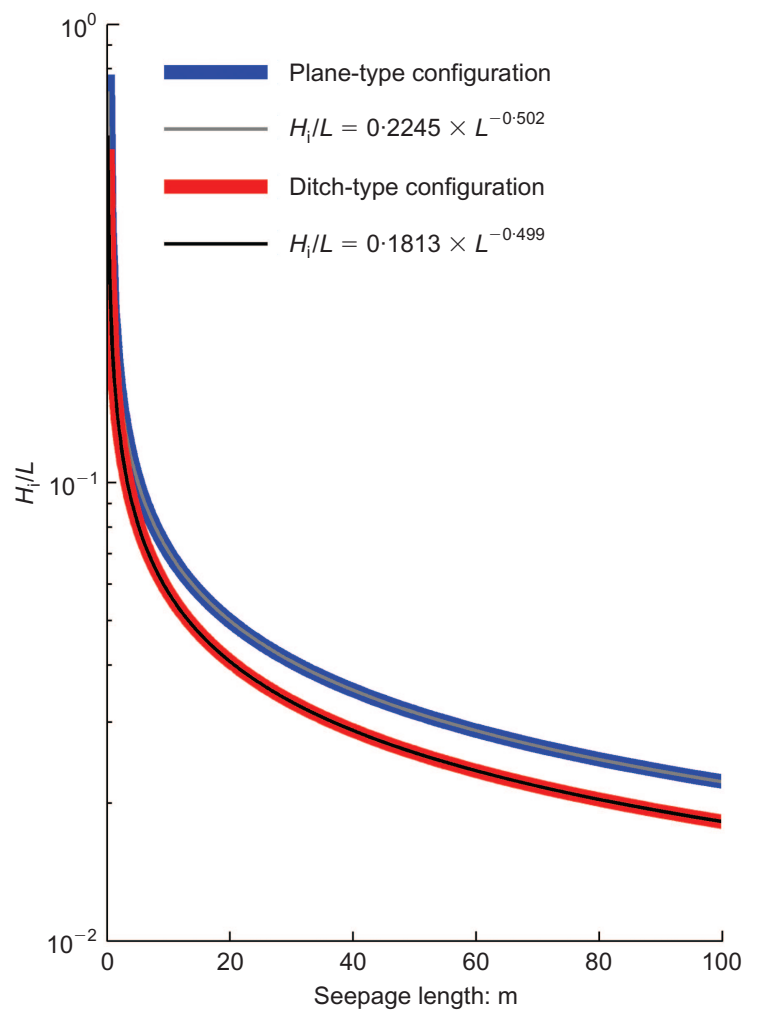

Fig. 10. Illustration of the scale effects of the initiation gradient for plane- and ditch-type configuration, and their least-square fits

because the group size for many experiments was found to be significantly larger than 20 grains. This is particularly the case with high-density sand samples, a finding which may be linked to inter-particle friction and interlocking, leading to the critical heave gradient $i_{\mathrm{c}}$ being larger than the values obtained with equations (1) and (2). Alternatively, it may be linked to the loosening of the bed with a fall in the exit gradient as a result. The difference in critical heave gradient for boiling and piping, as observed in some experiments and also by Rice \& Swainston-Fleshman (2013), merits more detailed investigation.

The model not only explains variations in the initiation gradient $H_{\mathrm{i}} / L$ due to scaling, it also clarifies the process of backward piping itself at different scales. Calculations with this model show that the initiation gradient $H_{\mathrm{i}} / L$ falls with

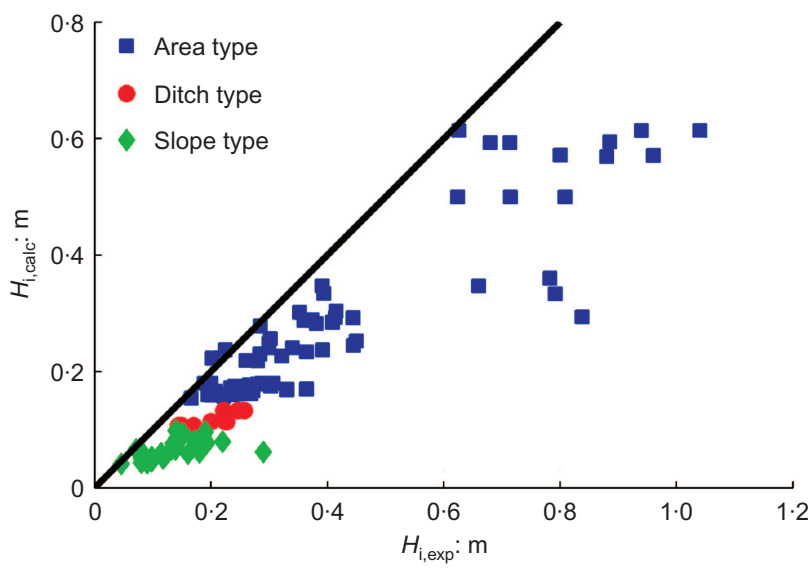

Fig. 11. Experimentally obtained and calculated critical gradients

the inverse square root of the seepage length $H_{\mathrm{i}} / L \propto 1 / \sqrt{L}$, assuming that other parameters, like grain size and ratio of thickness to length, remain constant. Progression of the pipe as predicted using the model of Sellmeijer (1988) is known to result in progression gradients that decrease with the inverse third-power root of the seepage length $\left(H_{\mathrm{p}} / L \propto 1 / \sqrt[3]{L}\right)$. This explains why initiation is more likely to dominate in small-scale experiments and progression in large-scale experiments. To study the progression of the pipe in small-scale experiments (which will remain necessary because costs increase rapidly with the size of the set-up), a different configuration should be used. For example, there should be a small, circular exit hole in which the concentration of flow lines causes initiation to occur at a relatively low gradient compared to the progression gradient (Van Beek et al., 2013). The understanding of the different processes and their scaling effects is essential to each experiment regardless of the scale of the experiment.

\section{CONCLUSIONS}

Backward erosion is governed by two processes: pipe initiation and progression. As the mechanisms of the processes are different, which of the two processes determines the critical head leading to failure depends on scale and configuration. Observations in experiments illustrate that, in some experiments, the head at which the pipe initiates $\left(H_{\mathrm{i}}\right)$ is equal to the critical head $\left(H_{\mathrm{c}}\right)$ at which the sample fails. In other experiments, after the initiation of the pipe, the 
head needs to be increased for the pipe to progress to the upstream side. The present study analysed experiments in which the initiation head equalled the critical head, or in which the initiation head was measured, to develop a model to predict pipe initiation.

The proposed model assumes the heave of a group of particles that causes the initiation of backward erosion. To validate this approach, relationships between the applied gradient $H / L$ and the exit gradient $i$ have been described and used to calculate the size of the group fluidised in each of the experiments. The group size obtained appears to be in the same range for experiments with different scales, configurations and grain sizes, with a minimum of 20 times the mean grain diameter. Equations have been given for a conservative prediction of the initiation of piping for two configurations (listed in Table 6).

The understanding of the processes governing initiation and progression is essential for the further analysis of the topic. The understanding of the scaling effects of both mechanisms is required for the design of experimental work and an understanding of observations in the field.

The equations can be used to predict the initiation head $H_{\mathrm{i}}$, but they are suitable for homogeneous samples only. Heterogeneity affecting the local flow pattern is likely to result in concentrated flows that will complicate the prediction of the initiation head. However, in the field, the approach could be useful for assessing the significance of a sand boil. A sand boil that emerges at a location where flow concentrates - such as an improperly closed borehole or at the end of a ditch that runs transverse to the embankment is likely to initiate at a much lower head than the head required for progression. However, a sand boil that emerges in a sandy area is likely to initiate at a head close to the head for progression, and should be considered a more serious threat.

\section{ACKNOWLEDGEMENTS}

The research is part of the 'Research and Development of Flood Defense Assessment Tools WTI2017' programme funded by Rijkswaterstaat (Centre for Water Management) on behalf of the Dutch Ministry of Infrastructure and the Environment.

\section{NOTATION}

$a$ size of the group of particles to be heaved expressed as number of particles

$D$ sand sample thickness

$d x$ absolute size of the group of particles to be heaved

$d_{50}$ grain diameter for which $50 \%$ of the sample (by weight) is finer

$H$ head drop across the sand sample or embankment

$H_{\mathrm{b}}$ minimum head drop across the sand sample or embankment at which sand boiling occurs

$H_{\mathrm{c}}$ critical head drop across the sand sample or embankment at which ongoing erosion occurs

$H_{\mathrm{i}} \quad$ minimum head drop across the sand sample or embankment at which the pipe is initiated

$H_{\mathrm{p}}$ minimum head drop across the sand sample or embankment at which the pipe progresses, assuming the presence of a short pipe

$h$ sand sample thickness

$i$ vertical exit gradient

$i_{\mathrm{c}}$ critical heave gradient

$K$ complete elliptic integral of the first kind over $m$

$k$ permeability coefficient

$L$ length of seepage

$m$ calculation parameter in equation (4)

$n$ porosity

$Q$ groundwater flow

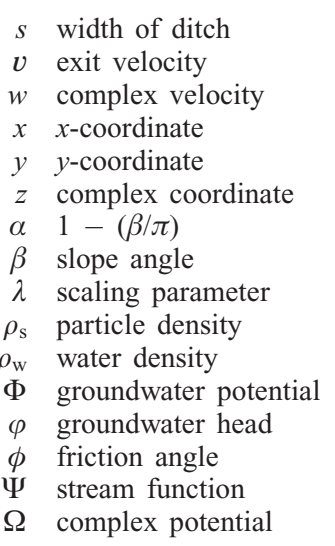

\section{REFERENCES}

Al Hattamleh, O., Razavi, M. \& Muhunthan, B. (2009). Experimental determination of representative elementary volume of sands using X-ray computed tomography. In Materials characterisation IV, WIT Trans. Engng Sci. 64 (eds. A. A. Mammoli and C. A. Brebbia), pp. 145-153. Ashurst, UK: WIT Press.

Bezuijen, A. \& Steedman, R. S. (2010). Scaling of hydraulic processes. In Proceedings of the 7th international conference on physical modeling in geotechnics (eds S. Springman, J. Laue and L. Seward). London, UK: Taylor and Francis Group.

Bligh, W. G. (1910). Dams, barrages and weirs on porous foundations. Engineering News 64, No. 26, 708-710.

Cao, D. (1994). Countermeasures for seepage erosion of Yangtze River main dikes. Yangtze River 25, No. 1, 25-30.

De Wit, J. M. (1984). Onderzoek zandmeevoerende wellen Rapportage Modelproeven, CO-220887/10. Delft, the Netherlands: Grondmechanica Delft (in Dutch).

ENW (Expertisenetwerk Waterveiligheid) (2010). Piping - realiteit of rekenfout. See http://www.enwinfo.nl/upload/Piping\%20-\%20 Realiteit\%20of\%20Rekenfout\%20(72dpi).pdf (accessed 16/09/ 2013; in Dutch).

Foster, M. A. \& Fell, R. (1999). A framework for estimating the probability of failure of embankment dams by piping using event tree methods, UNICIV Report No. R377. Sydney, Australia: School of Civil and Environmental Engineering, The University of New South Wales.

Foster, M., Fell, R. \& Spannagle, M. (2000). The statistics of embankment dam failures and accidents. Can. Geotech. J. 37, No. 5, 1000-1024.

GeoDelft (2002). MSeep user manual, release 6·7, November 2002. Delft, the Netherlands: GeoDelft

Hanses, U. (1985). Zur Mechanik der Entwicklung von Erosionskanälen in geschichtetem Untergrund unter Stauanlagen. Dissertation, Grundbauinstitut der Technischen Universität Berlin, Germany (in German).

ICOLD (International Commission on Large Dams) (2013). Internal erosion of existing dams, levees and dikes, and their foundations - Volume 1: Internal erosion processes and engineering assessment, Bulletin preprint 164. Paris, France: ICOLD.

Lane, E. W. (1935). Security from under-seepage masonry dams on earth foundations. Trans. Am. Soc. Civ. Engrs 100, No. 1, 929966.

Loezos, P. N., Costamagna, P. \& Sundaresan, S. (2002). The role of contact stresses and wall friction on fluidization. Chem. Engng Sci. 57, No. 24, 5123-5141.

Mansur, C. I., Postol, G. \& Salley, J. R. (2000). Performance of relief well systems along Mississippi river levees. J. Geotech. Geoenviron. Engng 126, No. 8, 727-738.

Miesel, D. (1978). Rückschreitende Erosion unter bindiger Deckschicht. In Baugrundtagung, pp. 599-626. Essen, Germany: Deutschen Gesellschaft für Erd-und Grundbau e.V. (in German).

Müller-Kirchenbauer, H., Rankl, M. \& Schlötzer, C. (1993). Mechanism for regressive erosion beneath dams and barrages. In Filters in geotechnical and hydraulic engineering (eds J. Brauns, M. Heibaum and U. Schuler), pp. 369-376. Rotterdam, the Netherlands: Balkema.

Pietrus, T. J. (1981). An experimental investigation of hydraulic 
piping in sand. Masters thesis, Department of Civil Engineering, University of Florida, Gainesville, FL, USA.

Poel, J. T. \& van der Schenkeveld, F. M. (1998). A preparation technique for very homogeneous sand samples and CPT research. In Proceedings of the international conference centrifuge (eds T. Kimura and O. Kusakabe), vol. 98, pp. 149-154. Rotterdam, the Netherlands: Balkema.

Polubarinova-Kochina, P. Y. A. (1962). Theory of groundwater movement. Princeton, NJ, USA: Princeton University Press.

Rice, J. \& Swainston-Fleshman, S. M. (2013). Laboratory modeling of critical hydraulic conditions for the initiation of piping. In Proceedings geo-congress 2013: Stability and performance of slopes and embankments III (eds C. Meehan, D. Pradel, M. A. Pando and J. F. Labuz), pp. 1044-1055. Reston, VA, USA: American Society of Civil Engineers (ASCE).

Richards, K. \& Reddy, K. R. (2007). Critical appraisal of piping phenomena in earth dams. Bull. Engng Geol. Environ. 66, No. 4, 381-402.

Richards, K. S. \& Reddy, K. R. (2012). Experimental investigation of initiation of backward erosion piping in soils. Géotechnique 62, No. 10, 933-942, http://dx.doi.org/10.1680/geot.11.P.058.

Schmertmann, J. H. (2000). The non-filter factor of safety against piping through sands. In Judgment and innovation (eds F. Silva and E. Kavazanjian), Geotechnical Special Publication No. 111, pp. 65-132. Reston, VA, USA: American Society of Civil Engineers (ASCE)

Sellmeijer, J. B. (1988). On the mechanism of piping under impervious structures. Doctoral dissertation, Technische Universiteit Delft, the Netherlands.

Sellmeijer, J. B. (2006). Numerical computation of seepage erosion below dams (piping). Proceedings of the 3rd international conference on scour and erosion, pp. 596-601. Gouda, the Netherlands: CURNET.

Sellmeijer, J. B., Lopéz de la Cruz, J., van Beek, V. M. \& Knoeff, J. G. (2011). Fine-tuning of the piping model through smallscale, medium-scale and IJkdijk experiments. Eur. J. Environ. Civ. Engng 15, No. 8, 1139-1154.
Silvis, F. (1991). Verificatie piping model: proeven in de deltagoot. Delft, the Netherlands: Grondmechanica Delft (in Dutch).

Skempton, A. W. \& Brogan, J. M. (1994). Experiments on piping in sandy gravels. Géotechnique 44, No. 3, 449-460, http:// dx.doi.org/10.1680/geot.1994.44.3.449.

TAW (Technische Adviescommissie voor de Waterkeringen) (1999). Technisch rapport Zandmeevoerende wellen. Delft, the Netherlands: Technische Adviescommissie voor de Waterkeringen (in Dutch).

Terzaghi, K. (1922). Der Grundbruch an Stauwerken und seine Verhüting (The failure of dams by piping and its prevention). Die Wasserkraft 17, No. 24, 445-449, reprinted in Terzaghi, K. (1960) From theory to practice in soil mechanics, pp. 114-118. New York, NY, USA: Wiley.

Townsend, F. C. D., Bloomquist, D., Shiau, J. M., Martinez, R. \& Rubin, H. (1988). Evaluation of filter criteria and thickness for mitigating piping in sand. Gainesville, FL, USA: Department of Civil Engineering, University of Florida.

Van Beek, M., van Knoeff, J. G. \& Sellmeijer, J. B. (2011). Observations on the process of backward piping by underseepage in cohesionless soils in small-, medium- and full-scale experiments. Eur. J. Environ. Civ. Engng 15, No. 8, 1115-1137.

Van Beek, M., van Bezuijen, A. \& Schenkeveld, F. M. (2012). Piping in loose sands - the importance of geometrical fixation of grains. Proceedings of the 2nd European conference on physical modelling in geotechnics (EUROFUGE 2012). Delft, the Netherlands: TU Delft.

Van Beek, M., van Bezuijen, A. \& Sellmeijer, H. (2013). Backward erosion piping. In Erosion in geomechanics applied to dams and levees (ed. S. Bonelli), pp. 193-269. London, UK and Hoboken, NJ, USA: Wiley.

Van Rhee, C. \& Bezuijen, A. (1992). Influence of seepage on stability of sandy slope. J. Geotech. Engng 118, No. 8, 1236-1240.

Weijers, J. B. A. \& Sellmeijer, J. B. (1993). A new model to deal with the piping mechanism. In Filters in geotechnical and hydraulic engineering (eds J. Brauns, U. Schuler and M. Heibaum), pp. 349-355. Rotterdam, the Netherlands: Balkema. 